\title{
Stiffness-Displacement Correlation from the RC Shear Wall Tests of the SAFE Program: Derivation of a Capacity Line Model
}

\author{
Francisco J. Molina, ${ }^{1}$ Pierre Pegon, ${ }^{1}$ and Pierre Labbé ${ }^{2}$ \\ ${ }^{1}$ European Commission, Joint Research Centre (JRC), Institute for the Protection and Security of the Citizen (IPSC), 21027 Ispra, Italy \\ ${ }^{2}$ Université Paris-Est, RENON (IRC-ESTP, IFSTTAR), IRC-ESTP, 28 avenue du Président Wilson, 94234 Cachan, France \\ Correspondence should be addressed to Francisco J. Molina; francisco.molina@jrc.ec.europa.eu
}

Received 24 September 2015; Accepted 21 March 2016

Academic Editor: Santiago Garcia-Granda

Copyright (c) 2016 Francisco J. Molina et al. This is an open access article distributed under the Creative Commons Attribution License, which permits unrestricted use, distribution, and reproduction in any medium, provided the original work is properly cited.

\begin{abstract}
The response of 13 reinforced concrete shear walls submitted to successive seismic tests has been postprocessed to produce time histories of secant stiffness and displacement oscillation amplitude. For every wall an envelope curve of displacement amplitude versus stiffness is identified which is fairly modelled by a straight line in double logarithmic scale. This relatively simple model, when used as a capacity line in combination with the demand response spectrum, is able to predict in an approximate manner the maximum response to the applied earthquakes. Moreover, the graphic representation of the demand spectrum and a unique model capacity line for a group of equal walls with different assumed design frequencies on them gives a visual interpretation of the different safety margins observed in the experiments for the respective walls. The same method allows as well constructing vulnerability curves for any design frequency or spectrum. Finally, the comparison of the different identified line models for the different walls allows us to assess the qualitative effect on the behaviour of parameters such as the reinforcement density or the added normal load.
\end{abstract}

\section{Introduction}

The seismic tests performed on reinforced concrete (RC) shear walls within the SAFE program were aimed at observing the effect of several parameters in the response. Particularly, as discussed by Labbé et al. [1], the study of the effect of the design frequency on the safety margin was one of the objectives. The current work takes advantage of those experimental results in order to derive a structural degradation model that potentially may be used for predicting in an approximate manner the maximum response of those walls to any given seismic excitation.

The description of the degradation in the structural behaviour as a function of the damage, and particularly by means of a reduction in the stiffness (or eigenfrequency) that is a function of the maximum performed displacement, is present in many studies and we will refer here just to a few of them that have especial common points with the current work.
In the study of Benedetti and Limongelli [2], shakingtable tests results on masonry buildings were used to plot "effective modal stiffness" values as a function of the displacement regarding single cycles in the response to successive earthquakes on the same specimen. They observed how for those structures the stiffness $K$ was dependent only on the maximum previously reached displacement $X$ at every cycle. By keeping in this representation only the envelope points of growing displacement, they constructed a model $K(X)$ that reproduced the experimental envelope curve. The model was based on the combination of brittle-elastic elements and elastoplastic elements and was defined by four parameters that were identified from the experimental results.

In the work of Brun et al. [3], results from the seismic tests on the RC shear walls of the SAFE program were reproduced by a finite element model based on the fixed smeared crack concept. The dynamic results of such finite element model to a series of excitations were used for representing the fundamental frequency associated with every level of 
maximum displacement reached. There, the fundamental frequency $f$ was derived from the secant stiffness of single cycles. After doing similar observations to the ones of Benedetti and Limongelli [2], this envelope curve $f(X)$ from the numerical simulations was substituted by a formula based on a combination of continuous functions and defined by eight parameters that were identified. Then, a simplified dynamic model was used to produce the time response of a wall by just step-by-step integration of the linear 1-DoF equation of motion, but with variable natural frequency at every step, decided from the recorded maximum displacement according to the previously obtained curve $f(X)$. The damping ratio for this simplified dynamic model was chosen fixed at $7 \%$ and considered as an average of the experimental values.

On the other hand, in the more recent work of Brun et al. [4], the finite element model based on fixed smeared crack that had been used to produce dynamic response was now used to produce static pushover curves from which to extract the secant stiffness and derive the fundamental frequency at every level of displacement. Moreover, the derived $f(X)$ curve for every wall was successfully compared with the equivalent envelope curve derived from the experimental results of the SAFE program. This time the simplified dynamic model was implemented by using directly the $f(X)$ function, either observed from the numerical pushover simulations (first case) or from the PsD tests for every wall (second case), without additional simplification by a formula and with the use of damping values derived from the experimental ones. The computed responses of the simplified model were similar between the two cases and also with the experimental ones for most of the studied walls.

In the current work, as in the mentioned studies, the variation of the stiffness with respect to the displacement level is observed from the experimental results. The special methods used for deriving an instant secant stiffness and displacement amplitude, as well as the rule for defining the envelope curve $K(X)$ or $f(X)$, are introduced and applied for all the tested walls of the SAFE program. Then, as a step forward with respect to other works, such curve is used as a capacity line that can be directly checked with the demand spectrum [5] in order to predict the maximum response to a given earthquake. Moreover, for every single tested wall, or for groups of them in some cases, the envelope curve is adjusted to a very simple straight line in double logarithmic scale, which is also used as the capacity line instead of the experimental curves. Interestingly, this simple model (characterised by only two parameters) is able to reproduce the order of magnitude of the maximum response to the tested inputs in most of the cases. It is also effective for producing vulnerability lines that give justification to the observations made by Labbé et al. [1] regarding the influence of the design frequency in the safety margin, for example. Moreover, this study gives one more graphical illustration of the influence of two main factors on the safety margin, which are, according to Labbé [6], the type of structural stiffness degradation and the shape of the demand spectrum at the performed frequencies.

\section{SAFE Program Data Processing}

2.1. Testing Campaign. As described in other publications [1, $3,4,7]$, the wall specimens of the SAFE program were 13 with names T01...T13 and they were all seismically tested in pure shear at the ELSA laboratory by means of the pseudodynamic (PsD) method, which is a hybrid testing technique by which the inertial forces are modelled numerically [8].

For all the walls, the length was

$$
L=3 \mathrm{~m}
$$

and the height was

$$
H=1.2 \mathrm{~m} .
$$

The main parameters with different value among the walls are displayed in Tables 1 and 2. The thickness of the walls was

$$
t_{\mathrm{T} i}=0.16 \mathrm{~m}
$$

for the first four specimens T01...T04 and

$$
t_{\mathrm{T} i}=0.2 \mathrm{~m}
$$

for the remaining ones T05 ‥T13. Consequently, the section of every wall was

$$
S_{\mathrm{T} i}=L t_{\mathrm{T} i} .
$$

Even though the concrete used was of two types with different actual capacities, the design of the walls assumed a conventional capacity value of

$$
f_{c 28}^{d}=30 \mathrm{MPa}
$$

corresponding to a design shear modulus of

$$
G_{d}=1.424 \cdot 10^{10} \mathrm{~Pa}
$$

for a Poisson's ratio 0.2 (according to Labbé et al. [1]), and the design stiffness

$$
K_{d \mathrm{~T} i}=\frac{G_{d} S_{\mathrm{T} i}}{H} .
$$

In the SAFE program, the design frequency $f_{d \mathrm{~T} i}$ was made a key parameter that was imposed with several values among the different walls in order to observe the effect of it in the response. As shown in Table 1, its value was

$$
f_{d \mathrm{~T} i}=4,8 \text { or } 12 \mathrm{~Hz},
$$

whereas the design damping ratio was adopted constant

$$
\xi_{d}=7 \%
$$

for all the walls.

Table 1 also contains the reinforcement density in horizontal $\rho_{h \mathrm{~T} i}$ and vertical $\rho_{v \mathrm{~T} i}$ directions. These are understood as nominal ratio of the steel section divided by the concrete section transversal to the bars. The conventional design 
TABLE 1: Parameters of tested walls I.

\begin{tabular}{|c|c|c|c|c|c|c|c|}
\hline Specimen $\mathrm{T} i$ & $\begin{array}{l}t_{\mathrm{T} i} \\
\mathrm{~m}\end{array}$ & $\begin{array}{l}S_{\mathrm{T} i} \\
\mathrm{~m}^{2} \\
\end{array}$ & $\begin{array}{c}K_{d \mathrm{~T} i} \\
\mathrm{GN} / \mathrm{m} \\
\end{array}$ & $\begin{array}{c}f_{d \mathrm{~T} i} \\
\mathrm{~Hz} \\
\end{array}$ & $\begin{array}{l}M_{\mathrm{T} i} \\
\text { ton }\end{array}$ & $\begin{array}{c}\rho_{h \mathrm{~T} i}^{\#} \\
\% \\
\end{array}$ & $\begin{array}{c}\rho_{v \mathrm{~T} i}^{\#} \\
\% \\
\end{array}$ \\
\hline Wall T01 & 0.16 & 0.48 & $\$$ & $\$$ & $\$$ & $0.8(0.770)$ & $0.8(0.770)$ \\
\hline Wall T02 & 0.16 & 0.48 & $\$$ & $\$$ & $\$$ & $0.8(0.770)$ & $0.8(0.770)$ \\
\hline Wall T03 & 0.16 & 0.48 & 5.7 & 4 & 9017.5 & $0.8(0.770)$ & $0.8(0.770)$ \\
\hline Wall T04 & 0.16 & 0.48 & 5.7 & 12 & 1001.9 & $0.8(0.785)$ & $0.8(0.785)$ \\
\hline Wall T05 & 0.2 & 0.6 & 7.12 & 8 & 2818 & $0.8(0.767)$ & $0.8(0.767)$ \\
\hline Wall T06 & 0.2 & 0.6 & 7.12 & 12 & 1252.4 & $0.6(0.628)$ & $0.4(0.402)$ \\
\hline Wall T07 & 0.2 & 0.6 & 7.12 & 4 & 11272 & $0.6(0.628)$ & $0.4(0.402)$ \\
\hline Wall T08 & 0.2 & 0.6 & 7.12 & 12 & 1252.4 & $0.4(0.402)$ & $0.4(0.402)$ \\
\hline Wall T09 & 0.2 & 0.6 & 7.12 & 4 & 11272 & $0.4(0.402)$ & $0.4(0.402)$ \\
\hline Wall T10 & 0.2 & 0.6 & 7.12 & 4 & 11272 & $0.6(0.628)$ & $0.6(0.628)$ \\
\hline Wall T11 & 0.2 & 0.6 & 7.12 & 4 & 11272 & $0.4(0.402)$ & $0.4(0.402)$ \\
\hline Wall T12 & 0.2 & 0.6 & 7.12 & 4 & 11272 & $0.11(0.113)$ & $0.11(0.113)$ \\
\hline Wall T13 & 0.2 & 0.6 & 7.12 & 12 & 1252.4 & $0.4(0.402)+\mathrm{CFRP}$ & $0.4(0.402)+$ CFRP \\
\hline
\end{tabular}

$\$$ The tests on walls T01 and T02 did not follow the same design scheme as the rest of the walls and their results will be used in this study only in a limited manner.

\# Actual values of reinforcement density implemented in the experiments are shown in brackets.

TABLE 2: Parameters of tested walls II.

\begin{tabular}{|c|c|c|c|c|c|c|c|}
\hline Specimen $\mathrm{T} i$ & $\begin{array}{l}\sigma_{n d T i} \\
\mathrm{MPa}\end{array}$ & $\begin{array}{c}\sigma_{n \mathrm{~T} i}(\text { actual }) \\
\mathrm{MPa}\end{array}$ & $\begin{array}{c}\tau_{d \mathrm{~T} i} \\
\mathrm{MPa}\end{array}$ & $\begin{array}{c}\gamma_{d \mathrm{~T} i} \\
\mathrm{mrad}\end{array}$ & $\begin{array}{l}u_{d \mathrm{~T} i} \\
\mathrm{~mm}\end{array}$ & $\begin{array}{c}{ }^{s} \operatorname{psa}_{0}\left(f_{d \mathrm{~T} i}, \xi_{d}\right) \\
\mathrm{m} / \mathrm{s}^{2}\end{array}$ & $\beta_{\mathrm{T} i}$ \\
\hline Wall T01 & 0 & 0.37 & 4.00 & 0.281 & 0.337 & $\$$ & $\$$ \\
\hline Wall T02 & 0 & 0.37 & 4.00 & 0.281 & 0.337 & $\$$ & $\$$ \\
\hline Wall T03 & 0 & 0.37 & 4.00 & 0.281 & 0.337 & 2.55 & 0.083 \\
\hline Wall T04 & 0 & 0.37 & 4.00 & 0.281 & 0.337 & 1.49 & 1.286 \\
\hline Wall T05 & 0 & 0.32 & 4.00 & 0.281 & 0.337 & 2.01 & 0.424 \\
\hline Wall T06 & 1 & 1.01 & 3.00 & 0.211 & 0.253 & 1.49 & 0.965 \\
\hline Wall T07 & 1 & 1.01 & 3.00 & 0.211 & 0.253 & 2.55 & 0.063 \\
\hline Wall T08 & 0 & 0.32 & 2.00 & 0.140 & 0.169 & 1.49 & 0.643 \\
\hline Wall T09 & 0 & 0.32 & 2.00 & 0.140 & 0.169 & 2.55 & 0.042 \\
\hline Wall T10 & 0 & 0.32 & 3.00 & 0.211 & 0.253 & 2.55 & 0.063 \\
\hline Wall T11 & 0 & 0.32 & 2.00 & 0.140 & 0.169 & 2.55 & 0.042 \\
\hline Wall T12 & 1 & 1.01 & 1.55 & 0.109 & 0.131 & 2.55 & 0.032 \\
\hline Wall T13 & 0 & 0.32 & 2.00 & 0.140 & 0.169 & 1.49 & 0.643 \\
\hline
\end{tabular}

${ }_{\$}$ The tests on walls T01 and T02 did not follow the same design scheme as the rest of the walls and their results will be used in this study only in a limited manner.

criterion applied to the SAFE walls [1] was based on the design shear stress defined as

$$
\tau_{d \mathrm{~T} i}=\rho_{v \mathrm{Ti}} f_{e d}+\sigma_{n d \mathrm{~T} i},
$$

where the nominal capacity of the steel was

$$
f_{e d}=500 \mathrm{MPa}
$$

and the nominal normal stress was

$$
\sigma_{n d \mathrm{Ti}}=0 \mathrm{MPa} \text { ("low") or } 1 \mathrm{MPa} \text { ("high") }
$$

depending on the wall as shown in Table 2. Note that the actual value of normal stress implemented in the tests was between 0.32 and 0.37 ("low") and 1.01 MPa ("high") but the design was based on the nominal values 0 or 1 . Similarly, for the reinforcement densities, the value entered in (11) was the nominal one, independently of its difference with the actual value also shown in Table 1 in brackets for completeness.

The associated design drift or shear deformation was

$$
\gamma_{d \mathrm{~T} i}=\frac{\tau_{d \mathrm{~T} i}}{G_{d}}
$$

and the design displacement at the top of the wall was

$$
u_{d \mathrm{~T} i}=H \gamma_{d \mathrm{~T} i} .
$$

The reference input motion $g_{0}(t)$ was an artificial accelerogram normalised to have Peak Ground Acceleration (PGA) of $1 \mathrm{~m} / \mathrm{s}^{2}$ (Figure 1). The sampling period of this specified accelerogram was

$$
\Delta t=0.01 \mathrm{~s}
$$




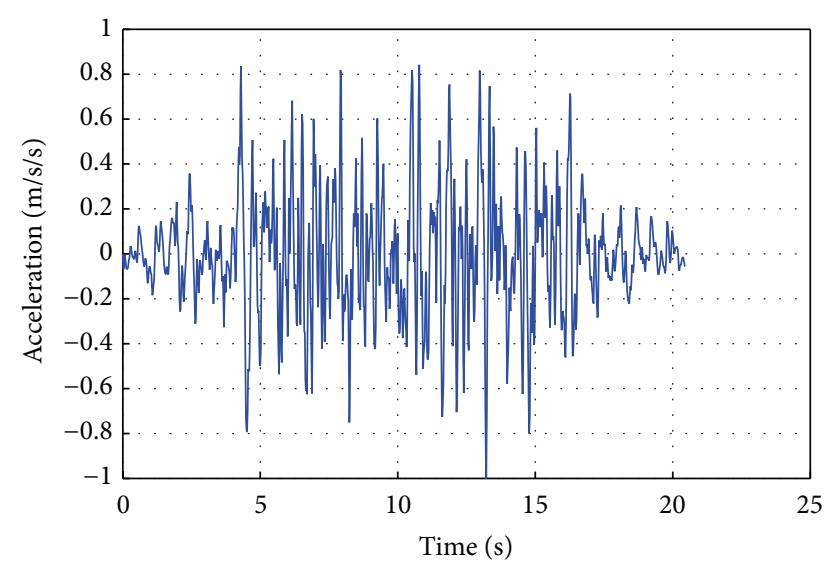

FIGURE 1: Reference input motion artificial normalised accelerogram $g_{0}(t)$.

Figure 2 shows the displacement response spectrum (RS) ${ }^{s} u_{0}(f, \xi)$ of the reference input and Figure 3 shows the associated pseudoacceleration spectrum

$$
{ }^{s} \operatorname{psa}_{0}(f, \xi)={ }^{s} u_{0}(f, \xi)(2 \pi f)^{2} .
$$

for damping ratio values of

$$
\xi=4,5,6,7 \% \text {. }
$$

The applied design criterion in the SAFE program was to match the design shear stress (11), and consequently associated drift (14) and displacement (15), at the design spectrum, for the design frequency and damping by introducing a scaling factor $\beta_{\mathrm{T} i}$; that is,

$$
u_{d \mathrm{~T} i}=\beta_{\mathrm{T} i}{ }^{s} u_{0}\left(f_{d \mathrm{~T} i}, \xi_{d}\right) .
$$

Thus, the scaling factor for every wall is defined as

$$
\beta_{\mathrm{T} i}=\frac{u_{d \mathrm{~T} i}}{{ }^{s} u_{0}\left(f_{d \mathrm{~T} i}, \xi_{d}\right)}=\frac{\tau_{d \mathrm{~T} i} H / G_{d}}{{ }^{s} u_{0}\left(f_{d \mathrm{~T} i}, \xi_{d}\right)}
$$

with values shown in Table 2. Correspondingly, the design input motion of wall $\mathrm{T} i$ is defined as

$$
g_{d \mathrm{~T} i}(t)=\beta_{\mathrm{Ti}} g_{0}(t)
$$

which nominally should induce the design stress in the wall.

Within the SAFE testing campaign, every wall was submitted to several successive earthquake runs with increasing intensity:

$$
g_{\mathrm{T} i j}(t)=\alpha_{\mathrm{T} i j} g_{d \mathrm{~T} i}(t),
$$

where $\alpha_{\text {Tij }}$ was the scaling factor of wall Ti for experiment run $j$ as displayed in Table 3.

The chosen design frequency for every wall was achieved by adopting a theoretical mass

$$
M_{\mathrm{T} i}=\frac{K_{d \mathrm{~T} i}}{\left(2 \pi f_{d \mathrm{~T} i}\right)^{2}}
$$

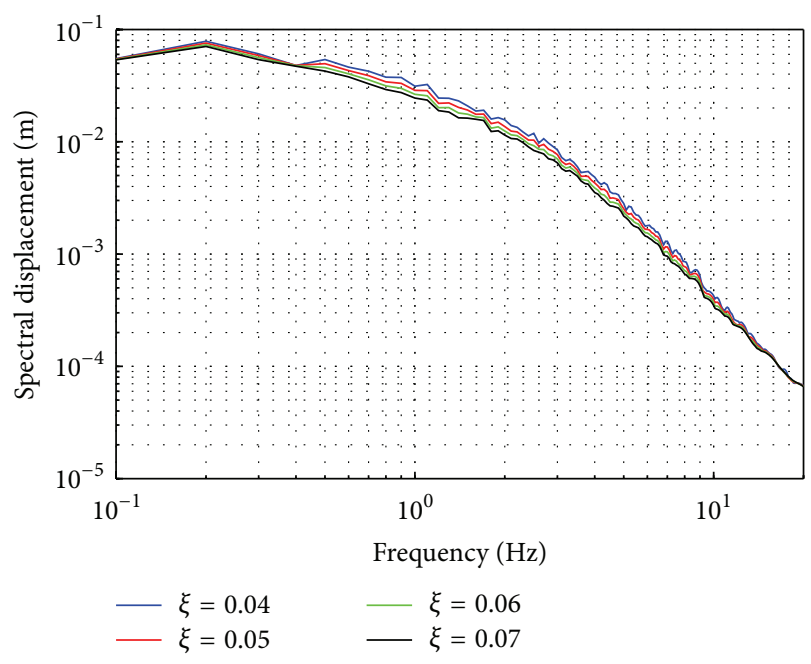

FIGURE 2: Displacement response spectrum ${ }^{s} u_{0}(f, \xi)$ of reference input.

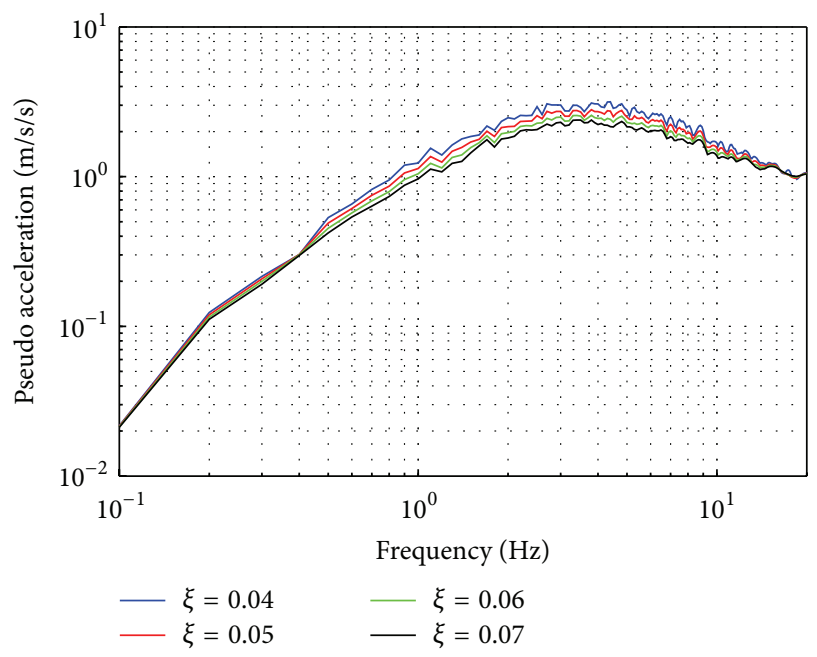

Figure 3: Pseudo acceleration response spectrum ${ }^{s} \operatorname{psa}_{0}(f, \xi)$ of reference input.

to oscillate with the displacement of the wall in the equation of motion integrated within the PsD testing method (Table 1).

Note that in Table 3, apart from the test name in the format "T $i-j$," a test reference code " $\mathrm{fNN}$ " is also shown for data traceability in the laboratory database. The last six columns in this table contain identified parameters that will be discussed in the following sections.

2.2. Signals Used for the Current Study. The current study is based on the identification of instantaneous values of secant stiffness, displacement amplitude, and other variables from the quasistatic pseudo-dynamic response at every experiment in order to characterise the behaviour of every wall specimen.

Within the applied PsD testing method, the step-bystep time-integrated displacement is sent to the controller of the actuators as a reference displacement that has to be reproduced at the feedback displacement transducer. This is 
TABle 3: Parameters of the performed tests.

\begin{tabular}{|c|c|c|c|c|c|c|c|c|c|c|c|}
\hline Wall & $\beta_{\mathrm{T} i}$ & Test & Test reference & $\alpha_{\mathrm{T} i j}$ & $\begin{array}{l}\text { PGA } \\
\mathrm{m} / \mathrm{s}^{2}\end{array}$ & $\begin{array}{l}u_{\mathrm{T} i j}^{*} \\
\mathrm{~mm}\end{array}$ & $\begin{array}{c}\bar{f}_{\mathrm{T} i j}^{*} \\
\mathrm{~Hz}\end{array}$ & $\begin{array}{c}\bar{\xi}_{\mathrm{T} i j}^{*} \\
\%\end{array}$ & $\begin{array}{l}\bar{G}_{\mathrm{T} i j}^{*} \\
\mathrm{GPa}\end{array}$ & $\begin{array}{c}\overline{\bar{\xi}}_{\mathrm{Ti}}\left(\bar{G}_{\mathrm{Tij}}^{*}\right) \\
\%\end{array}$ & $\begin{array}{c}\xi_{\mathrm{T} i}^{\mathrm{op}} \\
\% \\
\end{array}$ \\
\hline \multirow{2}{*}{ T01 } & \multirow{2}{*}{$\$$} & T01-1 & f03 & $\$$ & $\$$ & $\$$ & $\$$ & $\$$ & $\$$ & $\$$ & $\$$ \\
\hline & & T01-2 & f05 & $\$$ & $\$$ & $\$$ & $\$$ & $\$$ & $\$$ & $\$$ & $\$$ \\
\hline T02 & $\$$ & T02-1 & $\mathrm{f} 13$ & $\$$ & $\$$ & $\$$ & $\$$ & $\$$ & $\$$ & $\$$ & $\$$ \\
\hline \multirow{4}{*}{ T03 } & \multirow{4}{*}{0.083} & T03-1 & $\mathrm{f} 22$ & 1 & 0.0850 & 1.43 & 1.71 & 4.9 & 2.6144 & 4.7 & 5.3 \\
\hline & & T03-2 & $\mathrm{f} 23$ & 2 & 0.1700 & 4.67 & 1.27 & 4.7 & 1.4442 & 5.3 & 2.9 \\
\hline & & T03-3 & $\mathrm{f} 24$ & 3 & 0.2550 & 6.18 & 1.13 & 5.4 & 1.1438 & 5.5 & 6.0 \\
\hline & & T03-4 & $\mathrm{f} 25$ & 5 & 0.4250 & 11.74 & 0.86 & 15.2 & 0.6549 & 12.6 & 7.1 \\
\hline \multirow{3}{*}{ T04 } & \multirow{3}{*}{1.286} & T04-1 & $\mathrm{f} 32$ & 1 & 1.3061 & 8.06 & 3.74 & 4.6 & 1.3866 & 4.5 & 2.8 \\
\hline & & T04-2 & $\mathrm{f} 33$ & 1.3 & 1.6979 & 14.02 & 2.33 & 19.4 & 0.5383 & 24.2 & 9.6 \\
\hline & & T04-3 & $\mathrm{f} 34$ & 1.5 & 1.6470 & 12.13 & 1.71 & 14.9 & 0.2898 & 42.8 & 42.0 \\
\hline \multirow{5}{*}{ T05 } & \multirow{5}{*}{0.424} & T05-1 & $\mathrm{f50}$ & 1 & 0.4293 & 3.85 & 2.72 & 4.4 & 1.6480 & 5.0 & 5.5 \\
\hline & & T05-2 & $\mathrm{f} 51$ & 1.3 & 0.5581 & 6.50 & 2.51 & 5.1 & 1.4001 & 4.7 & 4.2 \\
\hline & & Т05-3 & $\mathrm{f} 52$ & 1.5 & 0.6438 & 7.18 & 2.37 & 4.5 & 1.2530 & 4.8 & 4.5 \\
\hline & & T05-4 & $\mathrm{f} 53$ & 2 & 0.8585 & 10.32 & 1.96 & 8.2 & 0.8561 & 8.4 & 7.0 \\
\hline & & T05-5 & f54 & 2 & 0.7216 & 18.63 & 0.88 & 14.9 & 0.1735 & 10.9 & 7.3 \\
\hline \multirow{4}{*}{ T06 } & \multirow{4}{*}{0.965} & T06-1 & $\mathrm{f} 44$ & 1 & 0.9797 & 3.10 & 4.27 & 4.5 & 1.8021 & 4.6 & 6.8 \\
\hline & & Т06-2 & $\mathrm{f} 45$ & 1.3 & 1.2736 & 6.22 & 3.52 & 5.6 & 1.2270 & 6.5 & 6.9 \\
\hline & & T06-3 & $\mathrm{f} 46$ & 1.5 & 1.4695 & 8.79 & 3.33 & 7.3 & 1.0972 & 7.0 & 5.9 \\
\hline & & T06-4 & $\mathrm{f} 47$ & 1.8 & 1.7634 & 14.43 & 2.37 & 13.4 & 0.5568 & 13.3 & 9.1 \\
\hline \multirow{4}{*}{ T07 } & \multirow{4}{*}{0.063} & Т07-1 & f58 & 1 & 0.0635 & 0.71 & 2.36 & 5.6 & 4.9715 & 5.7 & 4.6 \\
\hline & & Т07-2 & f59 & 2 & 0.1270 & 1.86 & 1.71 & 5.3 & 2.6030 & 5.3 & 7.8 \\
\hline & & Т07-3 & f60 & 5 & 0.3176 & 7.70 & 1.16 & 7.5 & 1.1956 & 7.3 & 4.6 \\
\hline & & Т07-4 & f61 & 10 & 0.6351 & 17.45 & 0.43 & 14.6 & 0.1629 & 17.0 & 8.0 \\
\hline \multirow{3}{*}{ T08 } & \multirow{3}{*}{0.643} & T08-1 & f65 & 1 & 0.6530 & 2.56 & 4.02 & 5.6 & 1.5975 & 5.6 & 6.0 \\
\hline & & Т08-2 & f66 & 1.4 & 0.9142 & 5.42 & 3.34 & 3.8 & 1.1052 & 4.8 & 5.9 \\
\hline & & T08-3 & $\mathrm{f} 67$ & 1.8 & 1.1754 & 11.85 & 1.80 & 13.1 & 0.3197 & 9.4 & 15.2 \\
\hline \multirow{4}{*}{ T09 } & \multirow{4}{*}{0.042} & T09-1 & f72 & 1 & 0.0423 & 0.58 & 2.34 & 5.4 & 4.8871 & 5.6 & 2.4 \\
\hline & & Т09-2 & f74 & 3 & 0.1268 & 2.25 & 1.51 & 5.0 & 2.0225 & 5.2 & 5.9 \\
\hline & & Т09-3 & f75 & 6 & 0.2536 & 5.74 & 1.15 & 4.2 & 1.1751 & 4.2 & 6.1 \\
\hline & & T09-4 & f76 & 10 & 0.4227 & 15.77 & 0.57 & 26.5 & 0.2864 & 28.7 & 8.0 \\
\hline \multirow{4}{*}{$\mathrm{T} 10$} & \multirow{4}{*}{0.063} & T10-1 & f84 & 1 & 0.0635 & 0.78 & 2.05 & 5.7 & 3.7418 & 6.0 & 6.1 \\
\hline & & T10-2 & f85 & 3 & 0.1905 & 5.43 & 1.27 & 5.0 & 1.4330 & 4.5 & 2.6 \\
\hline & & T10-3 & f86 & 6 & 0.3811 & 11.57 & 0.99 & 9.5 & 0.8742 & 8.3 & 4.3 \\
\hline & & T10-4 & f87 & 10 & 0.5339 & 14.72 & 0.78 & 16.1 & 0.5414 & 15.7 & 7.9 \\
\hline \multirow{3}{*}{ T11 } & \multirow{3}{*}{0.042} & T11-1 & f79 & 1 & 0.0423 & 0.46 & 2.36 & 3.8 & 4.9365 & 4.0 & 5.1 \\
\hline & & T11-2 & f80 & 6 & 0.2536 & 6.47 & 1.08 & 5.9 & 1.0302 & 6.0 & 5.6 \\
\hline & & T11-3 & $\mathrm{f} 81$ & 10 & 0.4227 & 13.38 & 0.75 & 9.0 & 0.4994 & 7.3 & 5.9 \\
\hline & & T12-1 & $\mathrm{f} 37$ & 1 & 0.0306 & 0.25 & 3.23 & 3.1 & 9.3013 & 3.5 & 2.9 \\
\hline & & T12-2 & $\mathrm{f} 38$ & 3 & 0.0917 & 0.85 & 2.19 & 5.0 & 4.2524 & 5.0 & 9.2 \\
\hline $\mathrm{T} 12$ & 0.032 & T12-3 & f39 & 5 & 0.1528 & 2.60 & 1.45 & 4.9 & 1.8587 & 5.2 & 6.8 \\
\hline & & $\mathrm{T} 12-4$ & $\mathrm{f} 40$ & 10 & 0.3056 & 7.80 & 1.03 & 7.0 & 0.9413 & 6.2 & 6.1 \\
\hline & & T12-5 & $\mathrm{f} 41$ & 15 & 0.4585 & 18.92 & 0.51 & 28.4 & 0.2328 & 29.2 & 6.9 \\
\hline & & T13-1 & $\mathrm{f} 90$ & 1 & 0.6530 & 0.40 & 8.83 & 4.2 & 7.7074 & 5.0 & 6.3 \\
\hline T13 & 0.643 & T13-2 & f91 & 1.8 & 1.1754 & 7.16 & 3.37 & 4.6 & 1.1242 & 6.0 & 5.4 \\
\hline & & T13-3 & $\mathrm{f} 92$ & 2.6 & 1.6977 & 15.58 & 1.35 & 12.1 & 0.1807 & 13.7 & 31.0 \\
\hline
\end{tabular}

\footnotetext{
${ }^{\$}$ The tests on walls T01 and T02 did not follow the same design scheme as the rest of the walls and their results will be used in this study only in a limited manner
} 


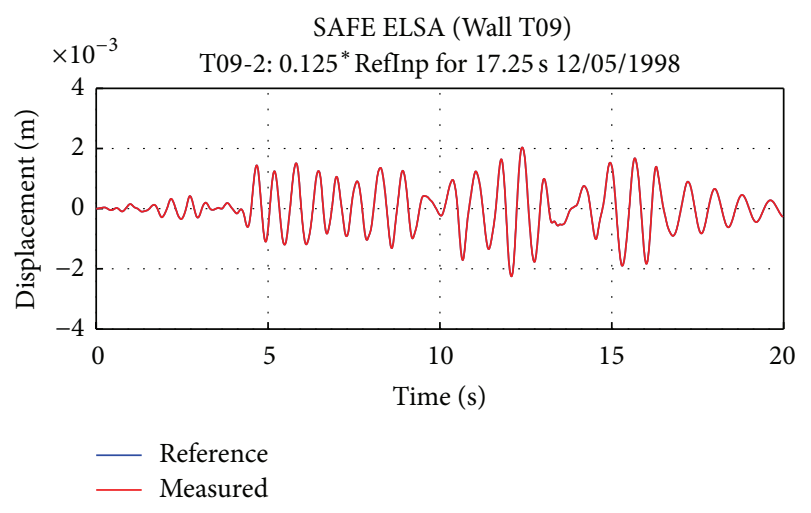

(a)

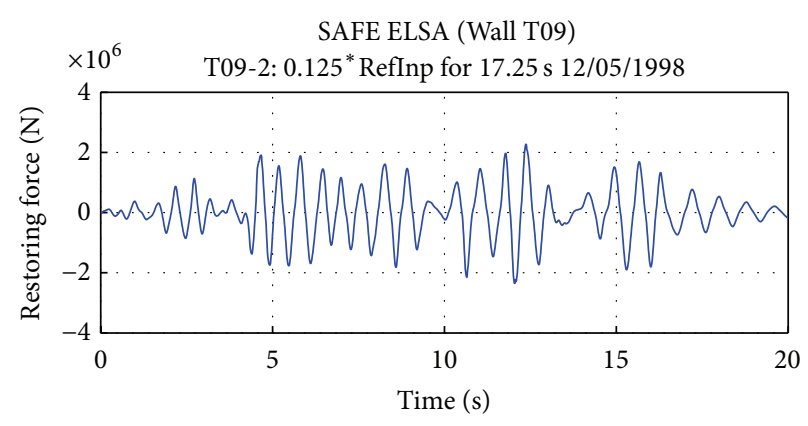

(b)

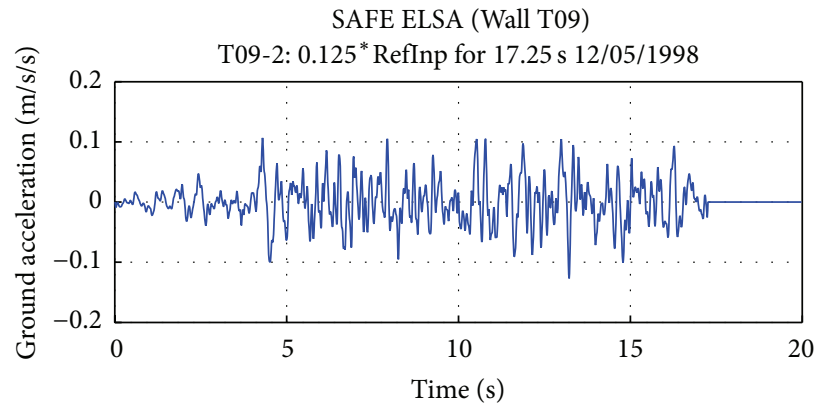

(c)

FIgURE 4: Example of earthquake response in the 2nd test on wall T09.

normally achieved with a small discrepancy which is called control error. In cases of inadequate testing conditions and, particularly for fast movement of the actuators, the amount of error may be large enough to affect significantly the apparent behaviour of the experimental model and its response to the specified input. This is due to the fact that the PsD equation of motion is based on measured forces that do not correspond to the integrated reference displacement $[9,10]$. This problem was present in the SAFE program for the tests on the first wall T01 and in a discrete manner on the second wall T02. However, even for those tests with an erroneous response to the earthquake, the synchronous measures of force and displacement always give reliable information about the real behaviour of the specimen as from any cyclic test, without considering the discrepant reference displacement at the controller. In addition, the tests performed on walls T01 and T02 did not follow the same method as the rest of the walls for the definition of the theoretical mass and the input acceleration. Thus, in this study, they will be considered only as cyclic tests and their results will be used only for the identification of the deformation-stiffness correlation.

As an example for a test response in one of the walls (wall T09, 2nd earthquake), in Figure 4, the time histories of reference and measured displacements (a), restoring force (b), and ground acceleration (c) are plotted. For most of the tests the input accelerogram was set to zero for a final period of time before the end of the test in order to observe the free response of the system. Note that for this experiment the accelerogram was set to zero from $17.25 \mathrm{~s}$ on. For the same wall T09, the measured force-displacement cycle for the four earthquakes applied to it is shown in Figure 5.

\subsection{Spatial Model for Frequency and Damping Identification.}

In contrast with other studies, such as the ones mentioned in the Introduction, the identification of stiffness (or eigenfrequency) and damping is not based here on a cycle-bycycle analysis. It is instead based on a moving observation window of constant duration. Nevertheless, the authors think that this choice would not affect much the proposed models and conclusions.

The Spatial Model method [9, 11-13] identifies the linearequivalent stiffness and damping coefficients that best fit the displacement and measured (quasistatic) restoring force. The identification is performed based, firstly, on the reference displacement and its derived velocity and, secondly, on the measured displacement and its derived velocity. In both cases the derivatives of the displacement are computed by central differences based on the recorded time increment that coincides with the sampling period of the accelerogram (16). In order to avoid the influence of the control errors in this study, the identification based on the measured displacement is used by default for further computation of parameters.

Since the linear identified model assumes a timeinvariant system (without degradation), the identification is repeated at every recording time increment of $0.01 \mathrm{~s}$ and is based on a window of data of limited duration that has constant length for all the earthquakes on the same specimen 


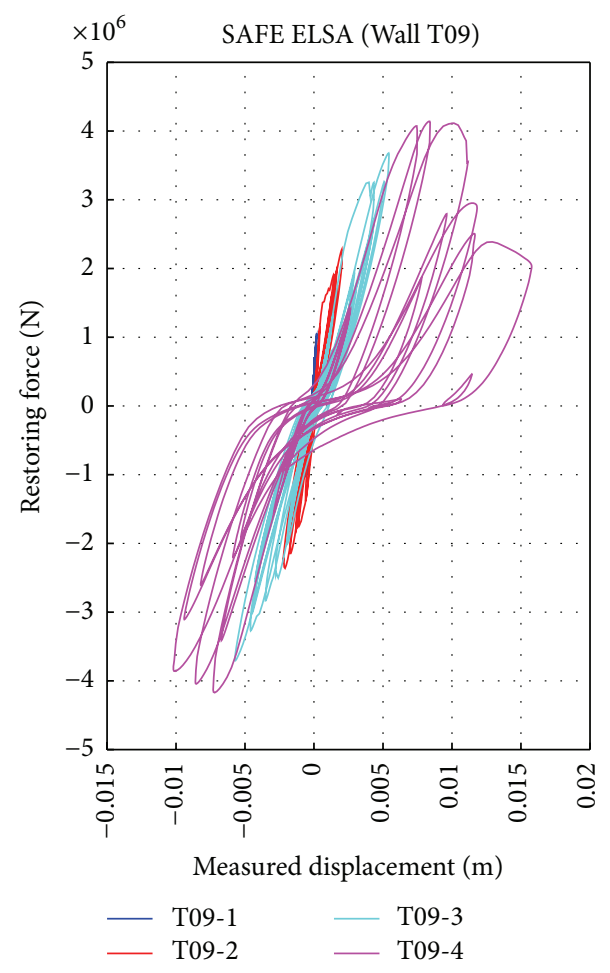

FIGURE 5: Force-displacement cycles for all the tests performed on wall T09.

Ti. That window is chosen to cover one "expected maximum" period of oscillation

$$
T_{\mathrm{T} i}^{\max }=2 \pi \sqrt{\frac{M_{\mathrm{T} i}}{\left(G^{\mathrm{min}} S_{\mathrm{Ti}} / H\right)}}
$$

or at least 40 time samplings $(0.4 \mathrm{~s})$, where it was assumed

$$
G^{\min }=5 \cdot 10^{8} \mathrm{~Pa} .
$$

Note that in general the adopted time window has to be narrow enough so that the system does not change strongly inside of it (due to degradation or change of oscillation amplitude), but, at the same time, it has to contain enough data to allow the compensation of different existing data noises. Owing to the width of the symmetric window around time $t$, this identification cannot be done centred at the beginning or the end of the record.

From the computed histories of identified stiffness $\bar{K}_{\mathrm{T} i}(t)$ and damping $\bar{C}_{\mathrm{T} i}(t)$, the eigenfrequency

$$
\bar{f}_{\mathrm{T} i}(t)=\frac{1}{2 \pi} \sqrt{\frac{\bar{K}_{\mathrm{T} i}(t)}{M_{\mathrm{T} i}}}
$$

and damping ratio

$$
\bar{\xi}_{\mathrm{T} i}(t)=\frac{\bar{C}_{\mathrm{T} i}(t)}{\sqrt{4 \bar{K}_{\mathrm{T} i}(t) M_{\mathrm{T} i}}}
$$

are derived based on the theoretical mass (23). The computed values, either from the reference displacement or from the measured displacement, are shown as an example for one of the tests in Figure 6. The comparison between both identified values allows us to know the magnitude of the effect of the control error on the identified parameters and presumably on the response. Such effect is negligible in the graphs within this figure.

The tendency of the frequency is to decrease with the time as a consequence of the larger amplitudes and the accumulated damage. On the other hand, the evolution of the damping is much more erratic and difficult to interpret.

2.4. Displacement Oscillation Amplitude. In this study, the instant value of identified stiffness or damping will be correlated with the amplitude of oscillation of the displacement rather than with the instant value of the displacement itself. To do so, the displacement amplitude is computed here by using the Hilbert Transform as computed through the Fast Fourier Transform (FFT) [14]. This technique has the advantage of being easy to automate. However, in the same way as for the identification of the stiffness and damping, a cycle-bycycle analysis approach could have been adopted instead.

For the identification of the displacement oscillation amplitude $\bar{u}_{\mathrm{T} i}(t)$, in order to have results that do not depend on the control errors, the measured displacement is used, unless stated otherwise. See the example of identified displacement amplitude in Figure 7(a).

Because the automatic identification of the displacement amplitude is based on the FFT, in cases in which the final amplitude was large in comparison to the initial one, it may give aberrant large amplitude values at the beginning of the record. This was avoided by adding a fictitious period of zeros (5s) at the end of the signals before applying the FFT. Then, back in the time domain, the computed amplitude was cut out the final $5 \mathrm{~s}$. This technique was used in a uniform manner for all the tests and was successful for avoiding the mentioned aberration, except for experiment T02-1. In fact this experiment was special because it showed very few oscillations before an extreme deformation was introduced collapsing the wall. In order to have a consistent amplitude curve, only for this experiment, a special exponential weighting window, starting just before the collapse of the specimen, was applied for the FFT.

Purely as a check, it is interesting to compare, on the one hand, the force amplitude derived from the identified stiffness multiplied by the displacement amplitude and, on the other hand, the real measured force history during the test. Such derived force amplitude (called projected amplitude in Figure 7(b)) appears to be a good approximation on the effective force amplitude, demonstrating the consistency of the identified stiffness as a secant stiffness.

2.5. Envelope of Drift Amplitude with respect to Shear Modulus. Before exploring the correlation between stiffness and displacement amplitude, another set of variables will be introduced for this study. In order to be able to compare walls of different length, thickness, and height or associated mass, for every earthquake, an identified equivalent shear modulus 


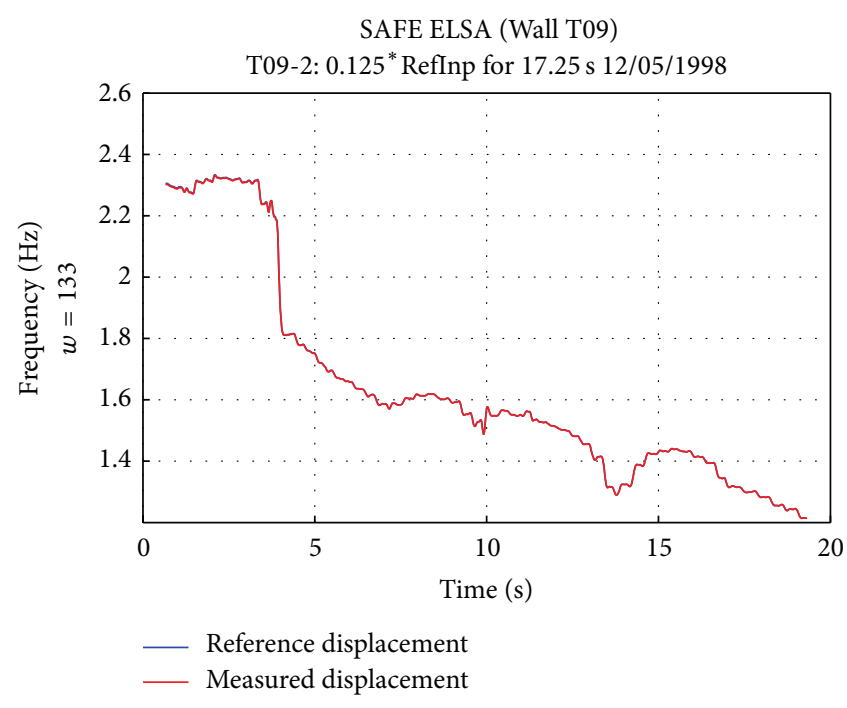

(a)

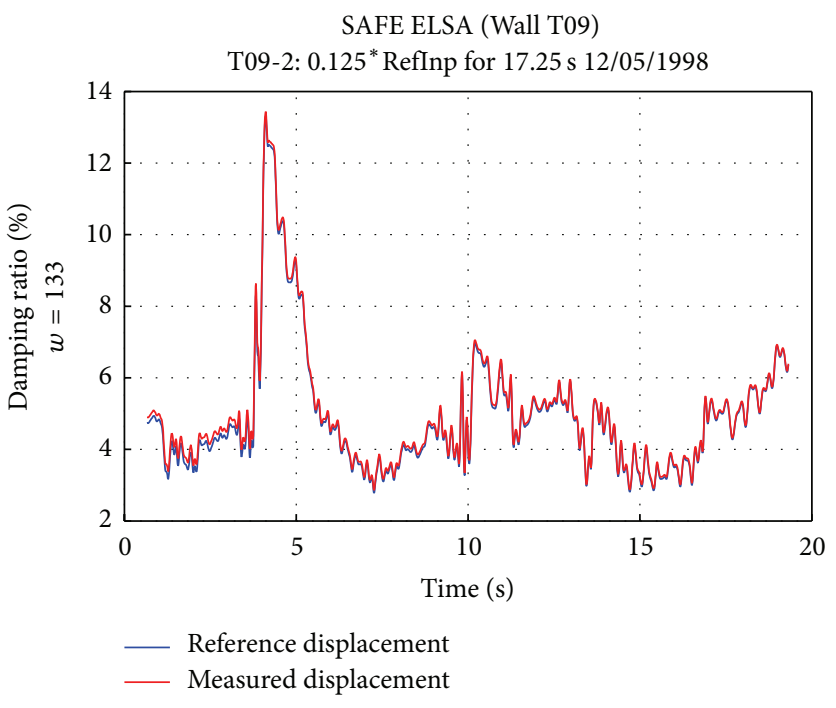

(b)

FIGURE 6: Example of identified frequency and damping ratio from 2nd earthquake response on wall T09. The number of sampling points used for the identification window was $w=133$ for this wall.

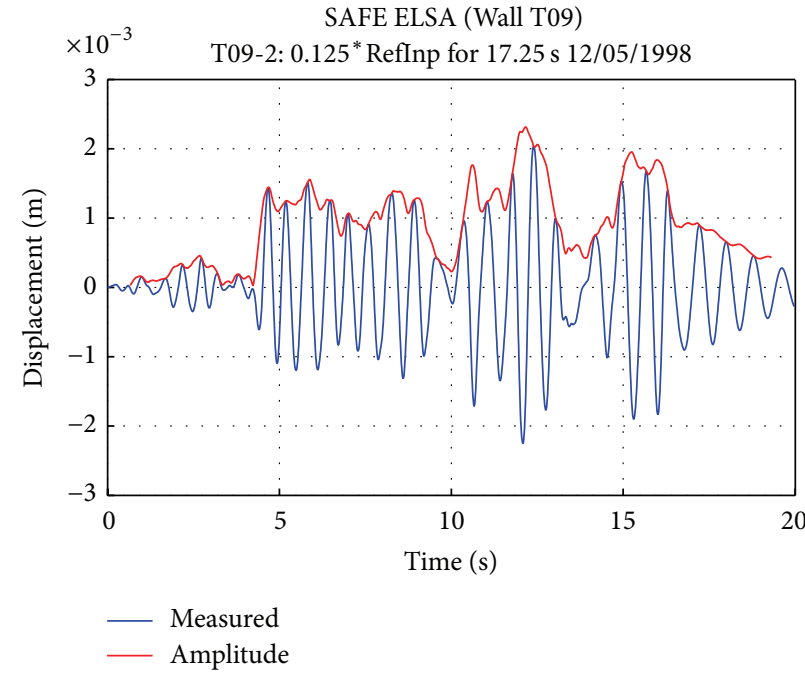

(a)

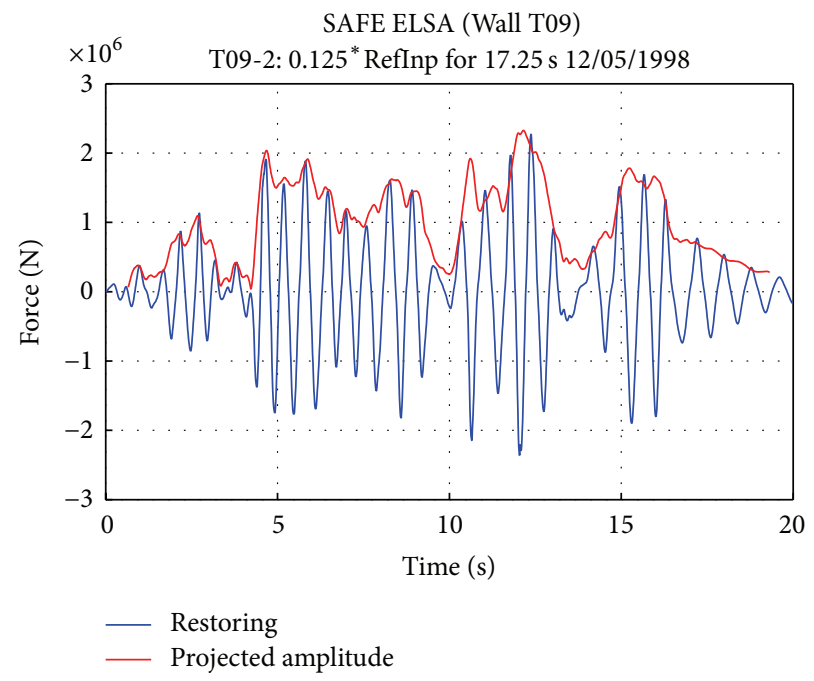

(b)

FIGURE 7: Example of identified displacement amplitude and projected force amplitude from 2nd earthquake response on wall T09.

history is derived from the stiffness (or squared frequency by mass) history, as identified by the Spatial Model, by dividing it by the section of the wall and multiplying by its height:

$$
\bar{G}_{\mathrm{Ti}}(t)=\frac{H}{S_{\mathrm{T} i}} \bar{K}_{\mathrm{T} i}(t)=\frac{M_{\mathrm{Ti}} H}{S_{\mathrm{T} i}}\left(2 \pi \bar{f}_{\mathrm{T} i}(t)\right)^{2} .
$$

Similarly, the identified amplitude of drift strain is simply computed as the amplitude of displacement divided by the height of the wall:

$$
\bar{\gamma}_{\mathrm{Ti}}(t)=\frac{\bar{u}_{\mathrm{Ti}}(t)}{H} .
$$

For all the earthquakes on a single wall, the synchronous values of $\bar{G}_{\mathrm{Ti}}(t)$ and $\bar{\gamma}_{\mathrm{T} i}(t)$ can be represented in the $(G, \gamma)$ axes. For example, this is done in double logarithmic scale in Figure 8(a) with a different colour for the response trajectory $\left(\bar{G}_{T i}(t), \bar{\gamma}_{T i}(t)\right)$ for every earthquake applied on wall T09 (tests T09-1 $\cdots 4$ in Table 3 ). For the progressively growing intensity input, there is a tendency to irreversibly decrease the identified equivalent shear modulus whenever the drift amplitude in the response reaches new higher values. The meaning of this is interpreted by assuming that new damage is introduced only when a new largest displacement amplitude is recorded. Then, a monotonic envelope line can be constructed by processing point by point chronologically from the successive trajectories but retaining only some selected points. More precisely, the first envelope point $t_{1}^{\times}$for the wall is selected as the first recorded point instant $t_{1}$ from its first 


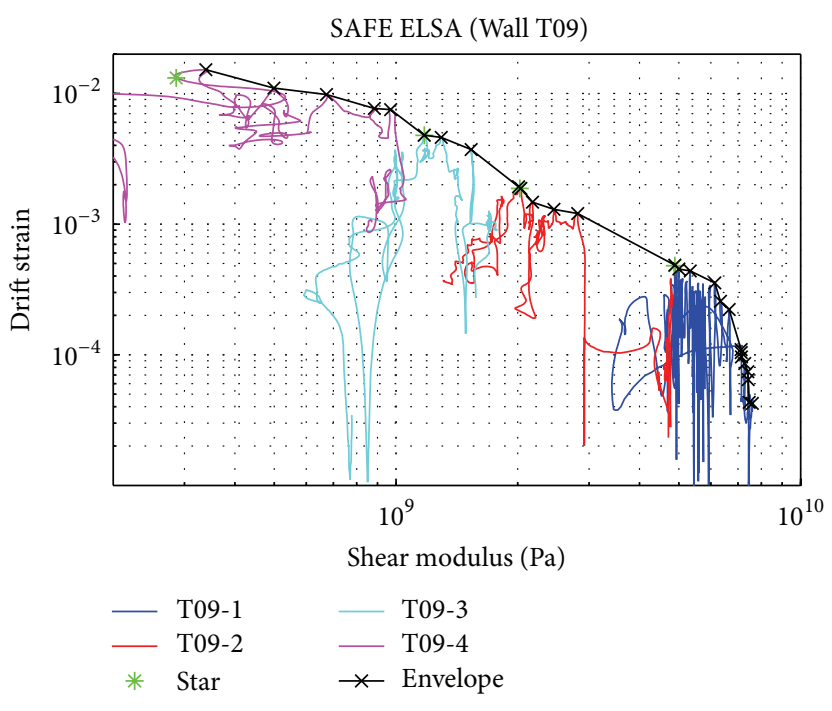

(a)

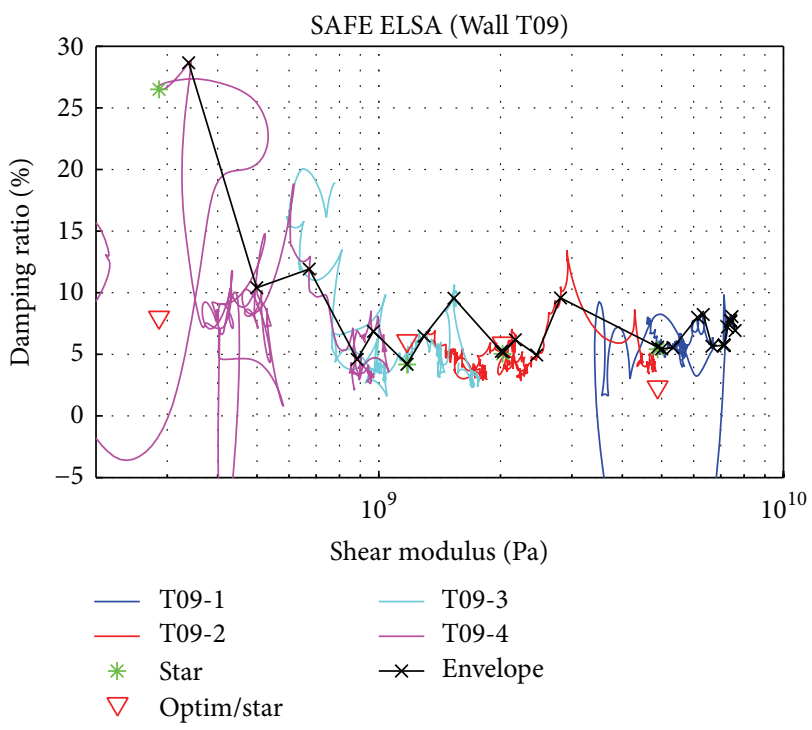

(b)

FIGURE 8: Identified values of drift amplitude and damping ratio as a function of the identified shear modulus from all the successive earthquake responses on wall T09.

test. Afterwards, a successive time instant is taken as a new selected point $t_{n+1}^{\times}$for the envelope only when, with respect to the last previous selected point $t_{n}^{\times}$, it represents a positive drift amplitude increment $\bar{\gamma}_{\mathrm{T} i}\left(t_{n+1}^{\times}\right)>\bar{\gamma}_{\mathrm{Ti}}\left(t_{n}^{\times}\right)$associated with a decrement of the modulus $\bar{G}_{\mathrm{T} i}\left(t_{n+1}^{\times}\right)<\bar{G}_{\mathrm{T} i}\left(t_{n}^{\times}\right)$. The obtained monotonic envelope is denoted by $\overline{\bar{\gamma}}_{\mathrm{Ti}}\left(\overline{\bar{G}}_{\mathrm{Ti}}\right)$ and in Figure 8 it is represented with a black line joining the selected points $\left(\bar{G}_{\mathrm{T} i}\left(t_{n}^{\times}\right), \bar{\gamma}_{\mathrm{Ti}}\left(t_{n}^{\times}\right)\right)$marked by crosses $\times$.

Note that there exist also cases of reversible decrease of the secant modulus associated with a decrease of the cycles amplitude. This is visible in Figure 8 and, by looking at Figure 5, it can be interpreted as a pinching behaviour associated with a gap at the central part of the cycles that is created by the previous existing cracks that close and reopen. Such decrease of the secant modulus is temporary: it does not seem to introduce new damage and does not contribute new points to the defined envelope.

In a similar manner, Figure 8 (b) shows in the $(G, \xi)$ axes the synchronous identified values of $\bar{G}_{\mathrm{Ti}}(t)$ and $\bar{\xi}_{\mathrm{Ti}}(t)$ for the same earthquakes in semilogarithmic scale. Then, considering only the time instants $t_{n}^{\times}$of the points that have been selected for the $\overline{\bar{\gamma}}_{\mathrm{T} i}\left(\overline{\bar{G}}_{\mathrm{Ti}}\right)$ envelope in Figure $8(\mathrm{a})$, the value of the viscous-equivalent damping ratio $\bar{\xi}_{\mathrm{Ti}}\left(t_{n}^{\times}\right)$allows creating a curve $\overline{\bar{\xi}}_{\mathrm{Ti}}\left(\overline{\bar{G}}_{\mathrm{Ti}}\right)$ of damping as a function of the modulus $\bar{G}_{\mathrm{T} i}\left(t_{n}^{\times}\right)$based on the contemporary points of the $\overline{\bar{\gamma}}_{\mathrm{Ti}}\left(\overline{\bar{G}}_{\mathrm{T} i}\right)$ envelope.

The maximum response absolute drift measured for every earthquake run $j$ on wall $\mathrm{T} i$

$$
\gamma_{\mathrm{T} i j}^{*}=\left|\gamma_{\mathrm{T} i}\left(t_{\mathrm{T} i j}^{*}\right)\right|
$$

happens at a time instant called $t_{\mathrm{T} i j}^{*}$ and can be represented in the same axes by associating with it a shear modulus

$$
\bar{G}_{\mathrm{T} i j}^{*}=\bar{G}_{\mathrm{T} i}\left(t_{\mathrm{T} i j}^{*}\right)
$$

and a damping ratio

$$
\bar{\xi}_{\mathrm{T} i j}^{*}=\bar{\xi}_{i}\left(t_{\mathrm{T} i j}^{*}\right)
$$

both taken to happen at the same time instant $t_{\mathrm{T} i j}^{*}$ as the maximum drift. These defined "star" values are represented in the same axes in Figure 8 with a green “*” mark for every earthquake run on wall T09. In general, these star points are close to the envelopes $\overline{\bar{\gamma}}_{\mathrm{T} i}\left(\overline{\bar{G}}_{\mathrm{T} i}\right)$ and $\overline{\bar{\xi}}_{\mathrm{T} i}\left(\overline{\bar{G}}_{\mathrm{T} i}\right)$ but do not fall exactly on them, because, firstly, they derive from the instantaneous absolute drift $\left|\gamma_{\mathrm{T} i}(t)\right|$ and not from the drift amplitude $\bar{\gamma}_{\mathrm{Ti}}(t)$ (see, e.g., the difference for the displacement in Figure 7) and, secondly, the values of shear modulus and damping correspond to different time instants from the ones selected for the envelope curves.

\section{Shear Wall Behaviour Modelling}

3.1. Interpretation of the Monotonic Envelope as a Capacity Curve. The value of the equivalent shear modulus at every moment can be assumed a function of the damage state in a wall, which is hypothetically considered a function of exclusively the maximum experienced drift amplitude in the specimen. With these assumptions, the previously derived monotonic envelope $\overline{\bar{\gamma}}_{\mathrm{T} i}\left(\overline{\bar{G}}_{\mathrm{T} i}\right)$ can be interpreted in general in Figure 9 as a capacity curve. For a given current value of equivalent shear modulus $G_{1}$ in a specimen, assuming 


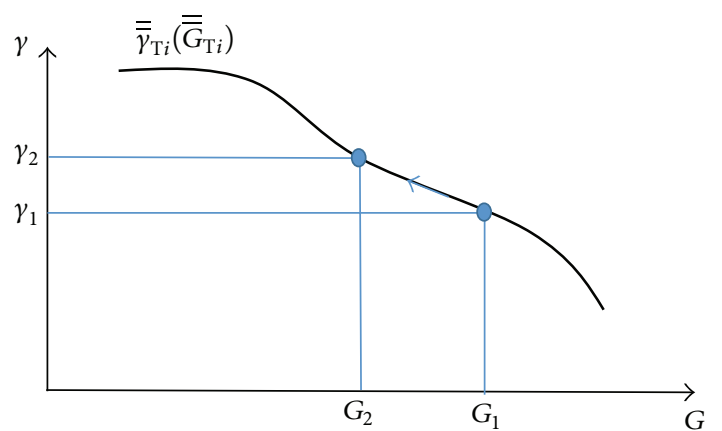

FIGURE 9: Interpretation of the monotonic envelope as a capacity curve.

constant stiffness behaviour for simplification, such capacity curve allows knowing the maximum value of drift deformation $\gamma_{1}=\overline{\bar{\gamma}}_{\mathrm{T} i}\left(G_{1}\right)$ that can be reached by that wall before introducing new damage in it. When that level of deformation $\gamma_{1}$ is exceeded up to a larger value $\gamma_{2}$, new damage is introduced allowing us to pass to higher values of strain corresponding to lower values of modulus in the capacity curve following from $\gamma_{1}=\overline{\bar{\gamma}}_{\mathrm{T} i}\left(G_{1}\right)$ up to $\gamma_{2}=\overline{\bar{\gamma}}_{\mathrm{Ti}}\left(G_{2}\right)$. After the introduction of this new damage, the current value of the modulus is $G_{2}$ and it will not change until a deformation larger than $\gamma_{2}$ is reached.

It must be noticed that the pinching effect is ignored by this modelling. The pinching effect was observed in the studied experiments and, as described in the previous section, is characterised by a temporary (mostly reversible) variation of the modulus associated with a reduction of the displacement oscillation amplitude. Because of such effects that make the secant stiffness change without introduction of new damage, the current concept of a capacity curve cannot be reliably used for predictions regarding deformation amplitudes smaller than the maximum one previously reached.

A further interpretation of such capacity curve model would be to use it for predicting the maximum response deformation to a given input accelerogram by means of the linear RS for a chosen value of damping ratio. According to this procedure, the estimated maximum response to that earthquake would be at the intersection point of the capacity curve $\overline{\bar{\gamma}}_{\mathrm{T} i}\left(\overline{\bar{G}}_{\mathrm{T} i}\right)$ with the RS represented in the axes $(G, \gamma)$. The concept of capacity curve is, in fact, the base for some existing design methods, even though the representation used here is different from the typical acceleration-displacement diagram [5]. Taking as a source the spectral displacement ${ }^{s} u_{\mathrm{T} i j}(f, \xi)$, in order to transform it to the current axes, these formulas are applied for every value of the frequency $f$ and displacement ${ }^{s} u_{\mathrm{T} i j}$ :

$$
\begin{aligned}
G & =\frac{H}{S_{\mathrm{T} i}} K_{\mathrm{T} i}=\frac{H}{S_{\mathrm{T} i}} M_{\mathrm{T} i}(2 \pi f)^{2}, \\
{ }^{s} \gamma_{\mathrm{T} i j} & =\frac{{ }^{s} u_{\mathrm{T} i j}}{H} .
\end{aligned}
$$

Note that, using (23) and (8), expression (33) can also be written as

$$
G=G_{d}\left(\frac{f}{f_{d \mathrm{~T} i}}\right)^{2} .
$$

The way to select the damping ratio for the computation of the RS is not established and, for research purposes in this section, we have used several values that result in several curves of the demand spectrum for a given earthquake to be checked against the capacity curve. In Figure 10 the RS for every earthquake is represented for three different values of damping ratio as follows:

(1) A design fixed value defined as $\xi_{d}=7 \%$ according to the design parameter (10).

(2) The interpolated value in the defined envelope damping line at $G=\bar{G}_{\mathrm{T} i j}^{*}$ : that is, $\overline{\bar{\xi}}_{\mathrm{T} i j}^{*}=\left.\overline{\bar{\xi}}_{\mathrm{Ti}}\left(\overline{\bar{G}}_{\mathrm{Ti}}\right)\right|_{\bar{G}_{\mathrm{T} i j}^{*}}$ (called $\xi_{\text {env }}$ in the graph legend), where $\bar{G}_{\mathrm{T} i j}^{*}$ is the modulus identified value at the time instant of the maximum experimental response (31). Point $\left(\bar{G}_{\mathrm{T} i j}^{*}, \overline{\bar{\xi}}_{\mathrm{T} i j}^{*}\right)$ is represented as a green "*” in Figure 8 (b) so that its value can be compared with the other values of damping.

(3) The optimal value $\xi_{\text {op }}$ that makes the value of the spectrum at $G=\bar{G}_{\mathrm{T} i j}^{*}$ coincide with the experimental maximum drift (30): that is, ${ }^{s} \gamma_{\mathrm{T} i j}\left(\bar{G}_{\mathrm{T} i j}^{*}, \xi_{\mathrm{op}}\right)=\gamma_{\mathrm{T} i j}^{*}$. This means that the spectrum is forced to pass exactly by the "star" point (green “*” in Figure 10) of the experimental maximum response for that test. Point $\left(\bar{G}_{\mathrm{T} i j}^{*}, \xi_{\mathrm{op}}\right)$ is represented as a red triangle in Figure $8(\mathrm{~b})$ so that its value can be compared with the other values of damping as well.

Finally, the square symbol in Figure 10 corresponds to the design point (design stress and design stiffness) from which the beta parameter (20) (fixing the intensity of the design earthquake) has been chosen so that the corresponding spectrum $(\alpha=1)$ passes through it at $G_{d}$. This point nominally corresponds to the response to the design earthquake, but typically it is not on the capacity curve because the effective initial stiffness of the wall is lower (in average by 0.7 according to Labbé et al. [1]) than the design one and because the design deformation (14) is typically reached for a certain level of damage with a degraded shear modulus. Note also that in some cases the design point might not be exactly on the response spectrum of any implemented test accelerogram, due to the difference regarding the applied duration of the signal (see the remarks done in Section 2.2).

3.2. Modelling the Monotonic Envelope by a Polygonal Curve. A generic experimental monotonic envelope of a wall linking drift deformation and shear modulus $\overline{\bar{\gamma}}_{\mathrm{Ti}}\left(\overline{\bar{G}}_{\mathrm{Ti}}\right)$, as introduced in the previous sections, is represented in double logarithmic scale in Figure 11 by using a black line. We propose here to model such envelope by means of a piecewise line curve. Starting from the lowest values of deformation, the model may include up to three straight-line segments as follows: 


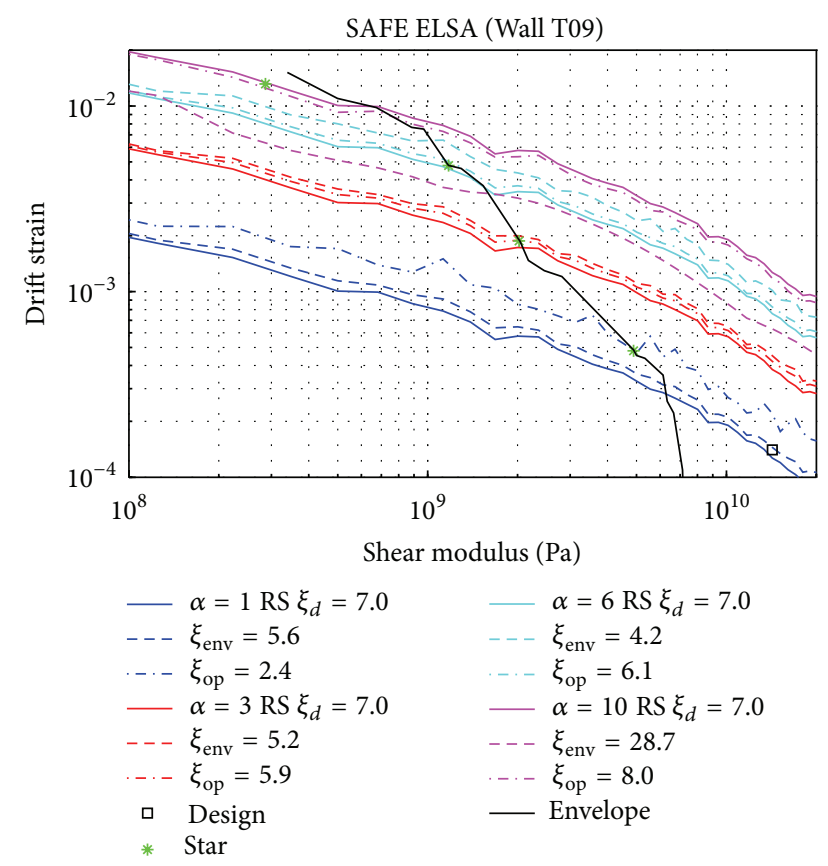

FIGURE 10: Matching of the monotonic envelope with the earthquake response spectra.

(i) Low Degradation Line, where the stiffness diminishes moderately when the deformation increases.

(ii) Regular Degradation Line (RDL), where the stiffness diminishes in a regular manner for a wide range of deformation amplitude (roughly for drift between $2 \cdot 10^{-4}$ and $\left.10^{-2} \mathrm{rad}\right)$.

(iii) Massive Degradation Line, where the stiffness accelerates its fall near the collapse of the wall.

The maximum response for most of the executed experiments in the SAFE program falls within the RDL segment, which is considered to extend between points $L$ and $M$ in Figure 11. The axis of the RDL is defined by the formula

$$
\frac{\gamma}{\gamma_{R}}=\left(\frac{G}{G_{R}}\right)^{m}
$$

which is a straight line in the double logarithmic scale that is defined by its slope $m$ and by passing by the reference point $R:\left(G_{R}, \gamma_{R}\right)$.

In the following sections this model will be identified for single walls or groups of walls and then it will be used as a simpler substitute of the envelope capacity line for predicting the maximum response to given earthquakes.

3.3. Polygonal Curve Model for Every Wall Specimen and Prediction of Maximum Response to Every Earthquake. In this section, from the monotonic envelope based on the experimental results on every wall, a polygonal curve model will be identified. For all the walls, the identification of the RDL

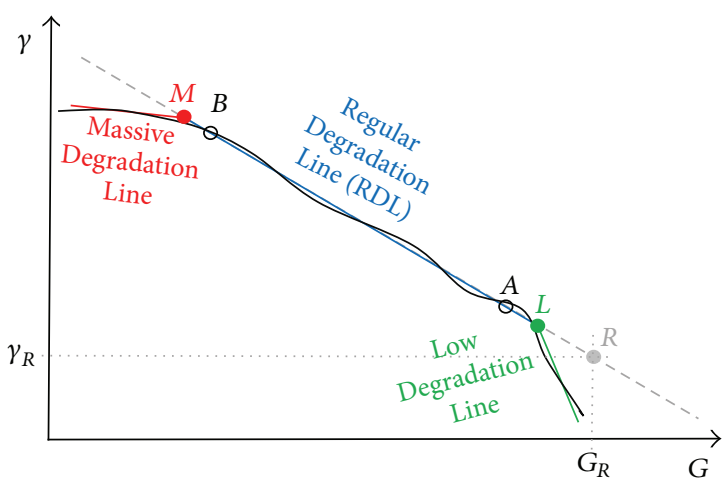

FIGURE 11: Piecewise line model of the monotonic envelope in double logarithmic scale.

segment, as represented in Figure 11 and (36), will be based on the envelope points for which two conditions apply:

$$
\begin{aligned}
& \gamma>2.5 \cdot 10^{-4}, \\
& G>7.0 \cdot 10^{8} \mathrm{~Pa}
\end{aligned}
$$

in order to make sure that the initial low degradation and final massive degradation phases are excluded. Since the abscissa of the reference point $R$ for the RDL axis is in principle arbitrary, here it has been chosen fixed as

$$
G_{R}=G_{d}=1.424 \cdot 10^{10} \mathrm{~Pa}
$$

so that for simplification it coincides with the design shear modulus (7). The coefficients in (36) have been identified after writing such formula in logarithmic form

$$
\log (\gamma)=m \log (G)-m \log \left(G_{R}\right)+\log \left(\gamma_{R}\right)
$$

and applying linear regression, using $\log (G)$ as independent variable and $\log (\gamma)$ as ordinate, taking the input values from the points of the experimental monotonic envelope $\overline{\bar{\gamma}}_{\mathrm{T} i}\left(\overline{\bar{G}}_{\mathrm{Ti}}\right)$. The identified parameters $m$ and $\gamma_{R}$ for every wall are collected in Table 4 .

In order to simplify the model as much as possible in the current application, we will assume that the abscissa of point $L$ (Figure 11), determining the start of the regular degradation in the model, equals the design shear modulus

$$
G_{L}=G_{d}=1.424 \cdot 10^{10} \mathrm{~Pa}
$$

and, thus, points $L$ and $R$ are coincident in this case. Note that a more accurate position of the abscissa of point $L$ could in practice be dependent on the casting method and manipulation of the specimen that always introduces an amount of initial damage. At the same time, the response to significant earthquakes typically falls rather in the regular degradation behaviour after the point $L$. Consequently, for practical reasons, the model does not pretend at this stage to be reliable regarding the position of the transition point $L$ between low and regular degradation and a fixed simple criterion has been chosen instead. The Low Degradation Line is chosen to be vertical with constant shear modulus. 
TABLE 4: For every wall specimen, identified parameters of RDL with maximum and standard errors of the model predictions by using the response spectrum of the applied earthquakes with damping ratios of $\xi=4,5,6$, or $7 \%$.

\begin{tabular}{|c|c|c|c|c|c|c|c|c|c|c|}
\hline Wall & $m$ & $\gamma_{R}(* 1 E-6)$ & $\begin{array}{c}\text { Max err\% } \\
(\xi=4 \%)\end{array}$ & $\begin{array}{c}\text { Max err\% } \\
(\xi=5 \%)\end{array}$ & $\begin{array}{c}\text { Max err\% } \\
(\xi=6 \%)\end{array}$ & $\begin{array}{c}\text { Max err\% } \\
(\xi=7 \%)\end{array}$ & $\begin{array}{l}\text { Std err\% } \\
(\xi=4 \%)\end{array}$ & $\begin{array}{l}\text { Std err\% } \\
(\xi=5 \%)\end{array}$ & $\begin{array}{l}\text { Std err\% } \\
(\xi=6 \%)\end{array}$ & $\begin{array}{l}\text { Std err\% } \\
(\xi=7 \%)\end{array}$ \\
\hline T01 & -1.7962 & 61.97 & $\$$ & $\$$ & $\$$ & $\$$ & $\$$ & $\$$ & $\$$ & $\$$ \\
\hline T02 & -1.7593 & 72.67 & $\$$ & $\$$ & $\$$ & $\$$ & $\$$ & $\$$ & $\$$ & $\$$ \\
\hline T03 & -1.7804 & 66.09 & 18 & 30 & 47 & 64 & 14 & 16 & 26 & 40 \\
\hline T04 & -1.8360 & 78.39 & 7 & 32 & 64 & 117 & 6 & 29 & 58 & 95 \\
\hline T05 & -1.7216 & 85.01 & 34 & 13 & 29 & 50 & 24 & 8 & 19 & 38 \\
\hline T06 & -1.5513 & 124.79 & 89 & 41 & 9 & 70 & 71 & 29 & 6 & 34 \\
\hline T07 & -1.6183 & 115.28 & 32 & 15 & 24 & 44 & 22 & 8 & 12 & 25 \\
\hline T08 & -1.6039 & 69.84 & 84 & 31 & 34 & 46 & 53 & 19 & 21 & 36 \\
\hline T09 & -1.6691 & 77.37 & 30 & 31 & 56 & 82 & 20 & 20 & 31 & 46 \\
\hline $\mathrm{T} 10$ & -1.7660 & 70.31 & 29 & 29 & 47 & 65 & 20 & 17 & 25 & 35 \\
\hline T11 & -1.7113 & 63.14 & 21 & 5 & 14 & 31 & 17 & 5 & 11 & 26 \\
\hline $\mathrm{T} 12$ & -1.4469 & 123.32 & 81 & $\begin{array}{c}84 \\
\left(57^{\#}\right)\end{array}$ & $\begin{array}{c}104 \\
\left(41^{\#}\right)\end{array}$ & $\begin{array}{c}124 \\
\left(57^{\#}\right)\end{array}$ & $\begin{array}{c}48 \\
\left(41^{\#}\right)\end{array}$ & $\begin{array}{c}45 \\
\left(32^{\#}\right)\end{array}$ & $\begin{array}{c}47 \\
\left(30^{\#}\right)\end{array}$ & $\begin{array}{c}53 \\
\left(34^{\#}\right)\end{array}$ \\
\hline \multirow[t]{2}{*}{$\mathrm{T} 13$} & -1.4699 & 142.31 & $\begin{array}{c}79 \\
\left(48^{\#}\right) \\
\end{array}$ & $\begin{array}{c}141 \\
\left(36^{\#}\right)\end{array}$ & $\begin{array}{c}147 \\
\left(46^{\#}\right)\end{array}$ & $\begin{array}{c}152 \\
\left(84^{\#}\right) \\
\end{array}$ & $\begin{array}{c}51 \\
\left(32^{\#}\right)\end{array}$ & $\begin{array}{c}66 \\
\left(19^{\#}\right) \\
\end{array}$ & $\begin{array}{c}71 \\
\left(28^{\#}\right) \\
\end{array}$ & $\begin{array}{c}92 \\
\left(57^{\#}\right) \\
\end{array}$ \\
\hline & & Average err: & $\begin{array}{c}46 \\
\left(43^{\#}\right)\end{array}$ & $\begin{array}{c}41 \\
\left(29^{\#}\right)\end{array}$ & $\begin{array}{c}52 \\
\left(37^{\#}\right)\end{array}$ & $\begin{array}{c}77 \\
\left(65^{\#}\right)\end{array}$ & $\begin{array}{c}31 \\
\left(29^{\#}\right)\end{array}$ & $\begin{array}{c}24 \\
\left(18^{\#}\right)\end{array}$ & $\begin{array}{c}30 \\
\left(24^{\#}\right) \\
\end{array}$ & $\begin{array}{c}47 \\
\left(42^{\#}\right) \\
\end{array}$ \\
\hline
\end{tabular}

$\$$ The tests on walls T01 and T02 did not follow the same design scheme as the rest of the walls and their results are used in this table only for the identification of the model and not for determination of the prediction error.

${ }^{\#}$ By using $G_{L}=1.0 \cdot 10^{10} \mathrm{~Pa}$, instead of $G_{L}=1.424 \cdot 10^{10} \mathrm{~Pa}$, in the model (see Section 3.8), the obtained error was different in the cases shown in brackets in this table.

Regarding point $M$ in (Figure 11) and the Massive Degradation Line, also a simple position is adopted by setting its abscissa at

$$
G_{M}=0
$$

since there is no high reproducibility in the observed behaviour in that phase and, anyway, it is hardly expected to be able to provide accurate predictions near the collapse. This means that the Regular Degradation Line (36) is assumed as the only model after point $L$ is overpassed (this simplifies the implementation of the model). Therefore, the current model cannot be reliable in the phases of low and massive degradation.

For example, in Figure 12 for Wall T09, the experimental monotonic envelope is represented by the thick yellow line and the obtained model is represented by the thick black line. The response spectra corresponding to the seismic experiments performed on that specimen are represented by different thin lines using damping ratio of $5 \%$ in (a) and $7 \%$ in (b). The figure shows the predicted maximum model response to every earthquake as the intersection of the spectrum with the model line (black rings). The same figure also shows the "star" points of maximum experimental response to the earthquake as introduced by (30).

In order to assess the reliability of the model predictions, its error has been quantified. The error of the model is defined here as the difference between the predicted maximum drift and the experimental one for the same earthquake. Such error has been computed in a special manner in Table 4 for every wall (walls T01 and T02 are not included in the computation of the error for the reasons explained in Section 2.2). The "maximum error" for every wall is obtained by computing the maximum, for the applied earthquakes, of the absolute difference between the logarithms of predicted and experimental drift. Alternatively, the "standard error" is obtained by computing the square root of the average, for the applied earthquakes, of the square of the difference between the logarithms of predicted and experimental drift. The derived maximum (or alternatively standard deviation) logarithm difference is then converted to error percentage by applying the antilogarithm, subtracting the unit and multiplying by 100 . The obtained error values in percentage are shown in the table for every wall and for four different cases of damping ratio used for the spectrum in the prediction $(\xi=4,5,6$ or $7 \%)$. In the last row of the table, among all the walls, the average value of every column is computed. The minimum value of such average is $41 \%$ for the maximum error (with $\xi=5 \%$ ) or $24 \%$ for the standard error (also with $\xi=5 \%)$ as shown in bold. This means that $\xi=5 \%$ is in general the most recommendable common value of damping ratio to introduce in the response spectrum in order to minimize, in the average, the prediction error regarding these experimental data and the applied model.

It should be mentioned that for the cases in which the experimental response of the wall was around $1 \%$ of drift, the behaviour entered in the massive degradation area of the envelope (Figure 11) and the model predictions for successive earthquakes were potentially unreliable. Due to this reason, in Table 4, the results from tests T04-3 and T05-5 (see Table 3) have not been included for the computation of the prediction 


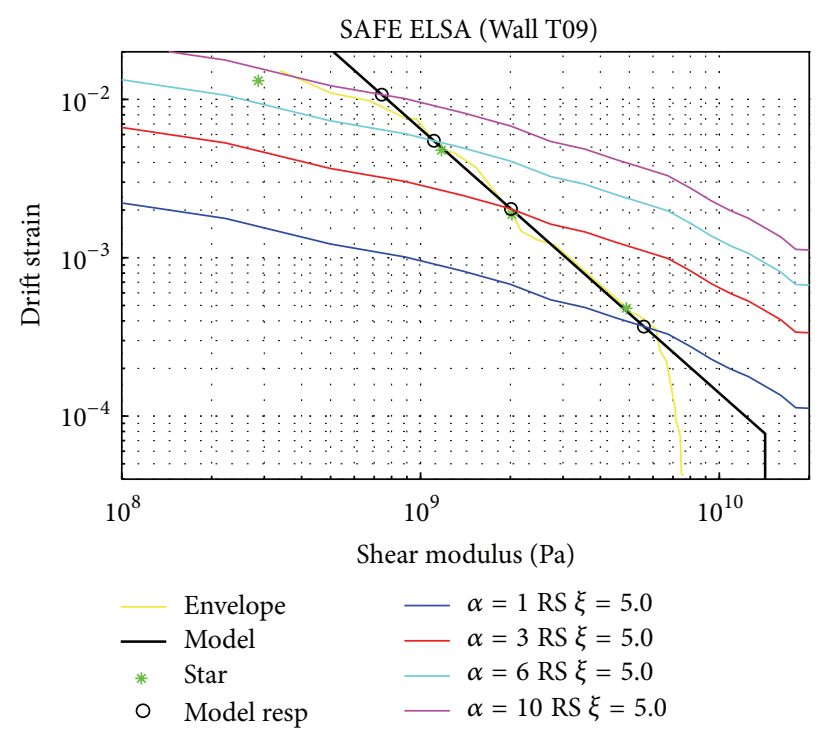

(a)

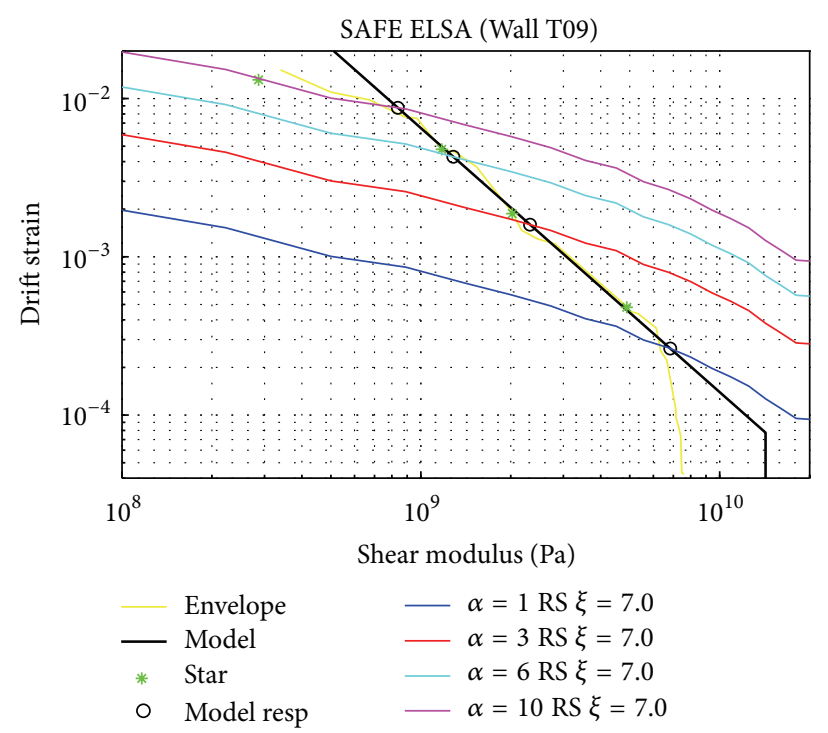

(b)

FIGURE 12: Piecewise line model of the monotonic envelope for Wall T09 and response spectra of the experimentally applied earthquakes for damping ratio, $\xi=5 \%$ (a) and $\xi=7 \%$ (b).

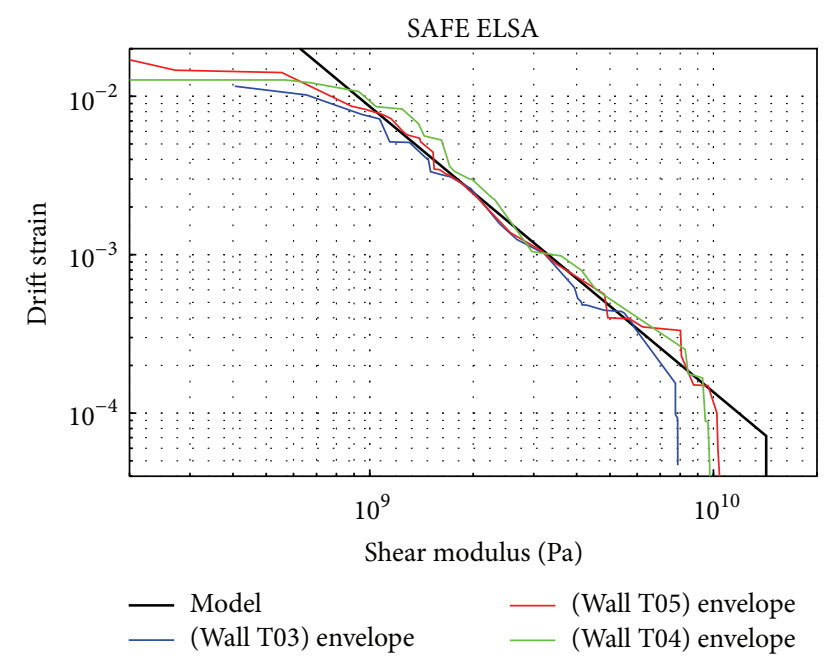

FIGURE 13: Identification of a unique model in the $(G, \gamma)$ axes by combining the data of walls T03, T04, and T05 (group " $\rho=0.8 r$ ").

error (even though they have been used for the identification of the model).

\subsection{Example of Model Identification from a Group of Walls and} Derivation of Respective Vulnerability Curves. In this section, the same definition of polygonal curve model of the previous section will be applied with (37) to (41), but using for the identification the experimental data from several walls at the same time, instead of from a single wall.

According to Tables 1 and 2, walls T03, T04, and T05 were constructed with (nominally) equal vertical and horizontal reinforcement parameters and were all tested with low vertical load. However, the first two of them had a thickness of $16 \mathrm{~cm}$ and the third one had a thickness of $20 \mathrm{~cm}$. Another difference in their tests was the specified design frequency $f_{d T i}$ which, respectively, was 4,12 , and $8 \mathrm{~Hz}$ as displayed in Table 1 . The experimental envelope in the $(G, \gamma)$ axes for the three walls is represented in Figure 13. In the same figure, the piecewise line model identified from the data of the three envelopes together is represented by a thicker black line with identified parameters:

$$
\begin{aligned}
& m=-1.8031, \\
& \gamma_{R}=7.181 \cdot 10^{-5} .
\end{aligned}
$$

Indeed, the degradation characteristics of the three walls are very similar in this representation, independently of the different thickness and specified design frequency for the tests. This suggests applying a unique model line for all of them. Moreover, their experimentally observed different behaviour in terms of safety margin can still be well explained by a unique model by considering their different implemented design frequencies. To do so, in Figure 14 the $(f, u)$ axes can be used instead of the ones used for the identification of the model in Figure 13. The conversion of the points of the model is done taking into account equations (34) and (35). Thus, the RDL definition by (36) transforms into

$$
\frac{u}{u_{R}}=\left(\frac{f}{f_{R}}\right)^{2 m}
$$

or equivalently

$$
\log (u)=2 m \log (f)-2 m \log \left(f_{R}\right)+\log \left(u_{R}\right)
$$




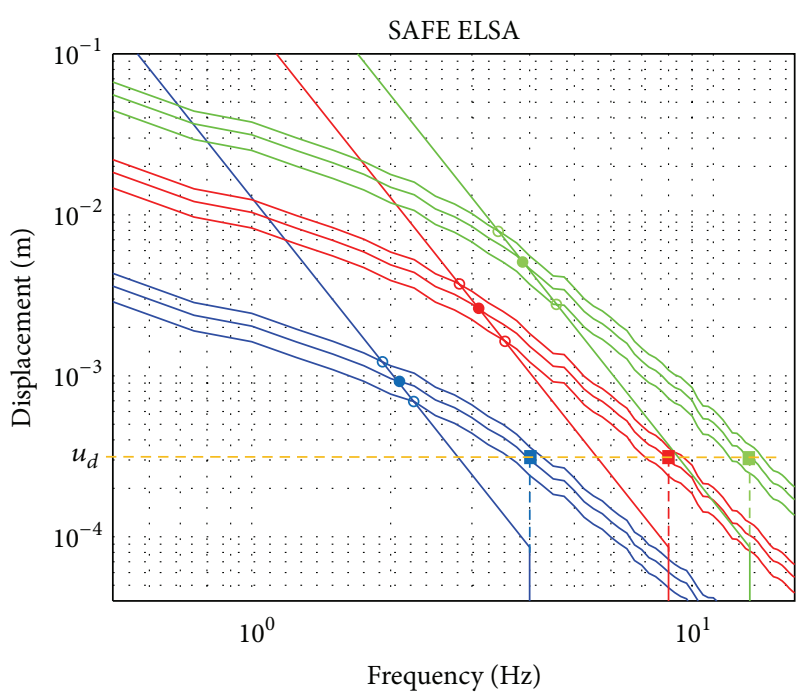

FIGURE 14: Representation of one unique model of the group of walls T03, T05, and T04 in the $(f, u)$ axes for the different respective design frequencies of 4 (blue), 8 (red), and $12 \mathrm{~Hz}$ (green). The response spectrum of the accelerogram is represented (with the respective colour) at the corresponding design intensity of every wall $\left(\mathrm{PGA}=\beta_{\mathrm{Ti}}\right)$ as well as for an increment and decrement of $20 \%$, with respect to that intensity.

with

$$
\begin{aligned}
& f_{L}=f_{R}=f_{d \mathrm{~T} i}\left(\frac{G_{R}}{G_{d}}\right)^{1 / 2}=f_{d \mathrm{~T} i}, \\
& u_{L}=u_{R}=H \gamma_{R}=1.2 \times 7.181 \cdot 10^{-5}=8.617 \cdot 10^{-5} \mathrm{~m}
\end{aligned}
$$

which is based on (38) and (42).

Thus, in Figure 14 one unique model based on parameters (42) appears as three response lines with corner point $f_{L}=$ $f_{d \mathrm{~T} i}$ at the design frequency for each one of the three walls, that is, $4 \mathrm{~Hz}$ (T03, blue line), $8 \mathrm{~Hz}$ (T05, red line), and $12 \mathrm{~Hz}$ (T04, green line). With the same respective colour for each wall, the response spectrum of the specified accelerogram at the respective design PGA is represented (for the design damping ratio of 7\%). More precisely, three lines are used for the input spectrum for every wall, the central one corresponding to the design level $\left(\mathrm{PGA}=\beta_{\mathrm{Ti}}\right)$, as indicated in Table 2, and the higher and lower ones corresponding to an increment and decrement of $20 \%$. This is done in order to emphasize graphically what the effect of a fixed percent variation of PGA can be on the predicted response. The design displacement for each wall was $u_{d}=3.37 \cdot 10^{-4} \mathrm{~m}$ according to Table 2, which corresponds to the value of the design spectrum at the design frequency for a damping ratio of $7 \%$. It is marked in the figure by a square symbol with the respective colours. However, the predicted displacement by using the piecewise line model is given by the intersection of the design spectrum with the RDL line of every wall and this is indicated by the solid circle in the figure. The predicted response for the incremented and decremented spectra is indicated by the ring symbols. In this manner, the representation done in Figure 14 allows us to interpret

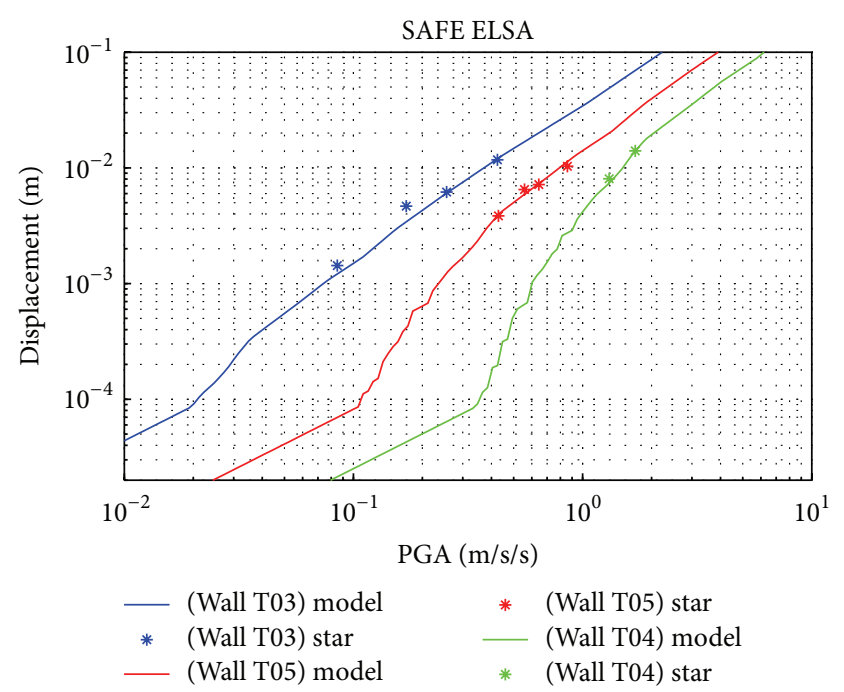

FIGURE 15: Predicted vulnerability line for walls T03, T04, and T05, according to one unique piecewise line model, in terms of displacement for every level of PGA based on the response spectrum for $5 \%$ damping ratio. Discrete ("star") points represent the recorded maximum experimental response at the applied earthquakes.

how several walls with the same mechanical characteristics may exhibit a clearly different behaviour depending on their design frequencies. In particular, two effects are explained here.

Firstly, since the design displacement parameter $u_{d}$ falls at a higher level than the model for the design frequency, the response to the design earthquake is predicted for a certain level of damage corresponding to a degraded frequency and thus at a higher displacement. This effect of increased response is inversely related to the difference in the global slopes of the RDL line and of the spectrum in the interval limited by the design frequency and the predicted degraded frequency. Thus, in the SAFE program, because the shape of the spectrum has steeper slope at higher frequencies, the higher the design frequency is, the higher the response displacement to the design earthquake becomes.

Secondly, for the represented fixed increment $(+20 \%$ and $-20 \%)$ of the intensity of the spectra, the amount of variation in the predicted response is different for every wall depending on its design frequency, being stronger for the case of higher design frequency in this study. This effect is again inversely related to the difference in the slopes between the RDL line and the spectrum, but now regarding only the local area around the predicted degraded frequency.

For different values of PGA, the predicted maximum response displacement is always obtained at the intersection of the spectrum with the piecewise line model. Thus, by applying this model, it is possible to derive the vulnerability curve of every wall for the specified accelerogram. The representation of the predicted displacement as a function of the imposed PGA is done in Figure 15 in log-log scale for every one of the three walls T03, T04, and T05. It must be observed that, for displacement values under $\sim 10^{-4} \mathrm{~m}$, the prediction falls in the low degradation constant-frequency 


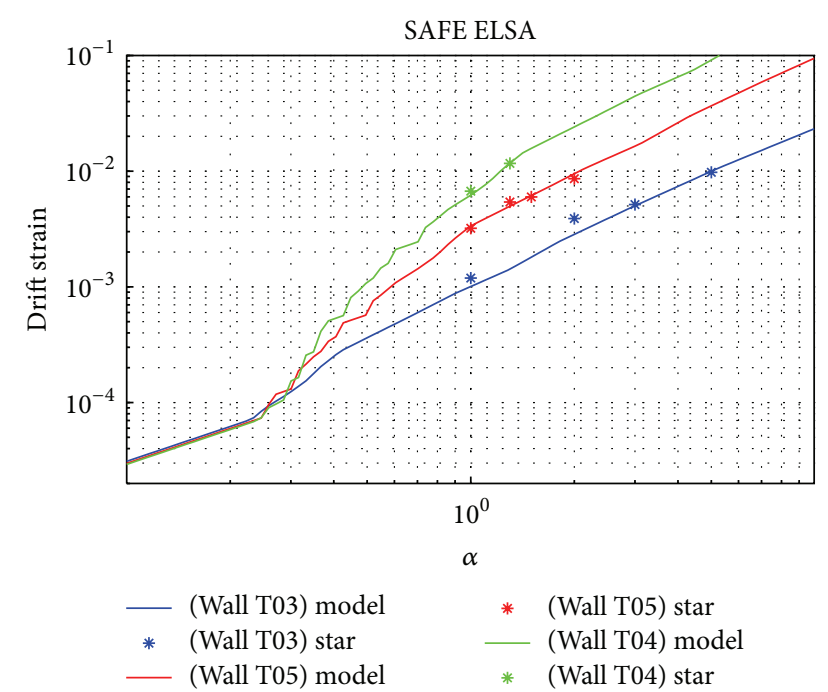

FIGURE 16: Predicted vulnerability line for walls T03, T04, and T05, according to one unique piecewise line model, in terms of drift for every level of design margin $\alpha$ based on the response spectrum for $5 \%$ damping ratio. Discrete ("star") points represent the recorded maximum experimental response at the applied earthquakes.

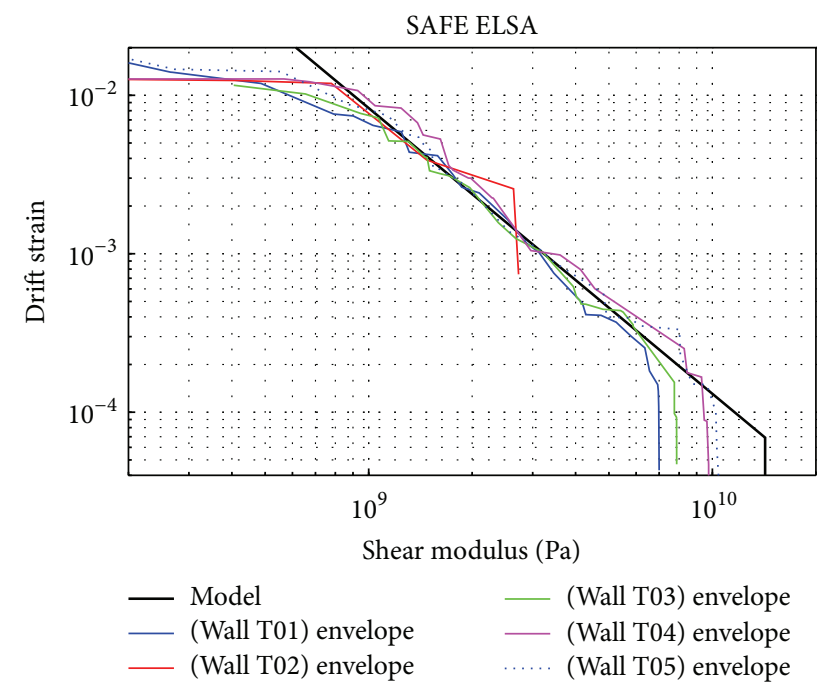

FIgURE 17: Identification of one unique model in the $(G, \gamma)$ axes by combining the data of walls T01 to T05 (group " $\rho=0.8$ ").

branch of the model that corresponds to a range with low reliability due to the large variability of results between the specimens. These vulnerability curves are derived in this occasion from the spectra for a damping ratio of $5 \%$ in order to minimize the prediction error. The " $*$ " symbols represented with the respective colour of every wall correspond to the experimental maximum response ("star" point as introduced by (30)) for every applied earthquake on the concerned wall.

Finally in Figure 16, the same vulnerability relationships (based on the response spectrum for 5\% damping ratio) and experimental data are represented but using the drift deformation and the design margin $\alpha$ (22) as variables in the axes. This representation shows again the two important effects of the change of the design frequency in this study that have been mentioned also for Figure 14: for higher design frequency, first, the larger response to the design earthquake (for $\alpha=1$ ) and, second, the successive larger relative increase of the response for every increase of $\alpha$.

It should be mentioned that, in Figures 15 and 16, the experimental "star" points for tests T04-3 and T05-5 are not represented since they took the walls to the total collapse and beyond the intended reliable range of applicability of the model.

Just for the purpose of completeness, even though T01 and T02 were not tested following the same criteria about the definition of the mass and the input, Figure 17 shows the experimental envelopes of these walls together with the ones already shown of T03, T04, and T05. All the walls in this figure had the same nominal reinforcement densities and low additional normal load. In this case, the piecewise line model identified from all the data of the five envelopes together is represented by the thick black line with identified parameters:

$$
\begin{aligned}
m & =-1.8032, \\
\gamma_{R} & =6.912 \cdot 10^{-5} .
\end{aligned}
$$

3.5. Model Identification for Different Groups of Walls. In the previous section, two models have been identified from two groups of walls. The current section is dedicated to perform similar identifications for a whole list of groups as shown in Table 5. In fact, the first two groups listed in this table are the ones described in the previous section with their respective identified parameters (42) and (46). As for Table 4, the current table also includes (for $\xi=4,5,6$, and 7\%) the values of the defined maximum and standard errors, which in this case are computed having into account all the experimental earthquakes applied to the respective group of walls (excluding T01 and T02). For the same reasons explained in the previous section, the results of tests T043 and T05-5 have not been used for the computation of the errors shown in Table 5.

As for Table 4, the columns referring to $\xi=5 \%$ have been shown in bold as well in Table 5 since it seems that this choice of damping, as a common parameter value, offers the best compromise for avoiding large errors.

The graphical representation of the models listed in Table 5 is done in Figures 13 and 17 (already discussed) and from Figures 18 to 23. These figures include the experimental envelopes of the affected walls and the single model line for the group identified by using the data of those envelopes. Finally, Figure 24 includes together just the identified models for the most representative groups from Table 5 .

3.6. Effect of Reinforcement and Normal Load on Characteristic Drift Values. In this section, the positions of the identified RDL line model (Figure 11) for all the walls will be compared in order to analyse the qualitative effects of the existing variability in reinforcement and normal load among the walls. To do so, instead of defining the RDL in terms of $m$ and $\gamma_{R}$ (with values displayed in Table 4), two 
TABLE 5: For every wall group, identified parameters of RDL with maximum and standard errors of the model predictions by using the response spectrum of the applied earthquakes with damping ratios of $\xi=4,5,6$, or $7 \%$.

\begin{tabular}{|c|c|c|c|c|c|c|c|c|c|c|c|}
\hline Group & Walls & $m$ & $\gamma_{R}(* 1 E-6)$ & $\begin{array}{c}\text { Max err\% } \\
(\xi=4 \%)\end{array}$ & $\begin{array}{c}\text { Max err\% } \\
(\xi=5 \%)\end{array}$ & $\begin{array}{c}\text { Max err\% } \\
(\xi=6 \%)\end{array}$ & $\begin{array}{c}\text { Max err\% } \\
(\xi=7 \%)\end{array}$ & $\begin{array}{l}\text { Std err\% } \\
(\xi=4 \%)\end{array}$ & $\begin{array}{l}\text { Std err\% } \\
(\xi=5 \%)\end{array}$ & $\begin{array}{l}\text { Std err\% } \\
(\xi=6 \%)\end{array}$ & $\begin{array}{l}\text { Std err\% } \\
(\xi=7 \%) \\
\end{array}$ \\
\hline$\rho=0.8$ & 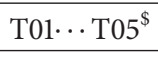 & -1.8032 & 69.12 & 34 & 35 & 52 & 70 & 21 & 13 & 23 & 40 \\
\hline$\rho=0.8 r$ & T03* T05 & -1.8031 & 71.81 & 32 & 37 & 54 & 73 & 19 & 13 & 26 & 44 \\
\hline $\begin{array}{l}\rho_{h}=0.6 \\
\rho_{v}=0.4\end{array}$ & $\begin{array}{l}\text { T06 } \\
\text { T07 } \\
\end{array}$ & -1.5865 & 119.65 & 81 & 25 & 24 & 76 & 44 & 14 & 9 & 35 \\
\hline$\rho=0.4$ & $\begin{array}{l}\text { T08 } \\
\text { T09 } \\
\text { T11 } \\
\end{array}$ & -1.6471 & 70.67 & 37 & 23 & 47 & 106 & 23 & 14 & 24 & 47 \\
\hline$\rho=0.6$ & $\mathrm{~T} 10$ & -1.7660 & 70.31 & 29 & 29 & 47 & 65 & 20 & 17 & 25 & 35 \\
\hline$\rho=0.11$ & $\mathrm{~T} 12$ & -1.4469 & 123.32 & 81 & $\begin{array}{c}84 \\
\left(57^{\#}\right) \\
\end{array}$ & $\begin{array}{c}104 \\
\left(41^{\#}\right) \\
\end{array}$ & $\begin{array}{c}124 \\
\left(57^{\#}\right)\end{array}$ & $\begin{array}{c}48 \\
\left(41^{\#}\right)\end{array}$ & $\begin{array}{c}45 \\
\left(32^{\#}\right)\end{array}$ & $\begin{array}{c}47 \\
\left(30^{\#}\right)\end{array}$ & $\begin{array}{c}53 \\
\left(34^{\#}\right)\end{array}$ \\
\hline CFRP & $\mathrm{T} 13$ & -1.4699 & 142.31 & $\begin{array}{c}79 \\
\left(48^{\#}\right) \\
\end{array}$ & $\begin{array}{c}141 \\
\left(36^{\#}\right)\end{array}$ & $\begin{array}{c}147 \\
\left(46^{\#}\right)\end{array}$ & $\begin{array}{c}152 \\
\left(84^{\#}\right)\end{array}$ & $\begin{array}{c}51 \\
\left(32^{\#}\right)\end{array}$ & $\begin{array}{c}66 \\
\left(19^{\#}\right)\end{array}$ & $\begin{array}{c}71 \\
\left(28^{\#}\right) \\
\end{array}$ & $\begin{array}{c}92 \\
\left(57^{\#}\right) \\
\end{array}$ \\
\hline ALL & $\mathrm{T} 01 \cdots \mathrm{T} 3^{\$}$ & -1.6360 & 90.82 & 368 & 226 & 158 & 324 & 49 & 37 & 39 & $\begin{array}{c}52 \\
\left(50^{\#}\right)\end{array}$ \\
\hline
\end{tabular}

${ }^{\$}$ The tests on walls T01 and T02 did not follow the same design scheme as the rest of the walls and their results are used in this table only for the identification of the model and not for determination of the prediction error.

${ }^{\#}$ By using $G_{L}=1.0 \cdot 10^{10} \mathrm{~Pa}$, instead of $G_{L}=1.424 \cdot 10^{10} \mathrm{~Pa}$, in the model (see Section 3.8), the obtained error was different in the cases shown in brackets in this table.

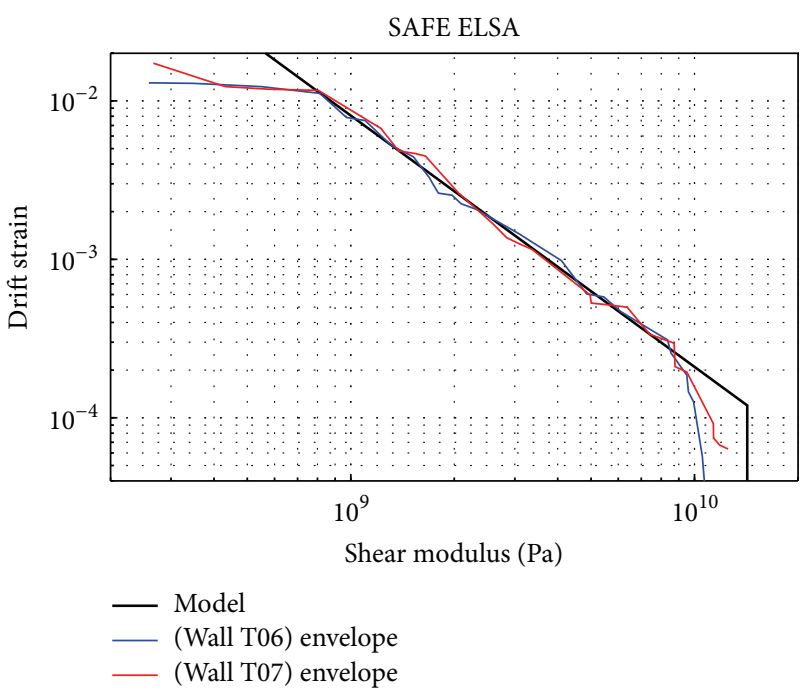

FIGURE 18: Identification of a unique model by including the data of walls T06 and T07 (group " $\rho_{h}=0.6, \rho_{v}=0.4$ ”).

alternative parameters with an easier physical interpretation will be introduced. The new parameters of the model will be the ordinates of the characteristic points $A$ and $B$ that are shown in Figure 11, that is, respectively, as given by (36),

$$
\gamma_{A}=\gamma_{R}\left(\frac{G_{A}}{G_{R}}\right)^{m}
$$

which is the drift capacity at a reference "initial" modulus $G_{A}$ and

$$
\gamma_{B}=\gamma_{R}\left(\frac{G_{B}}{G_{R}}\right)^{m}
$$

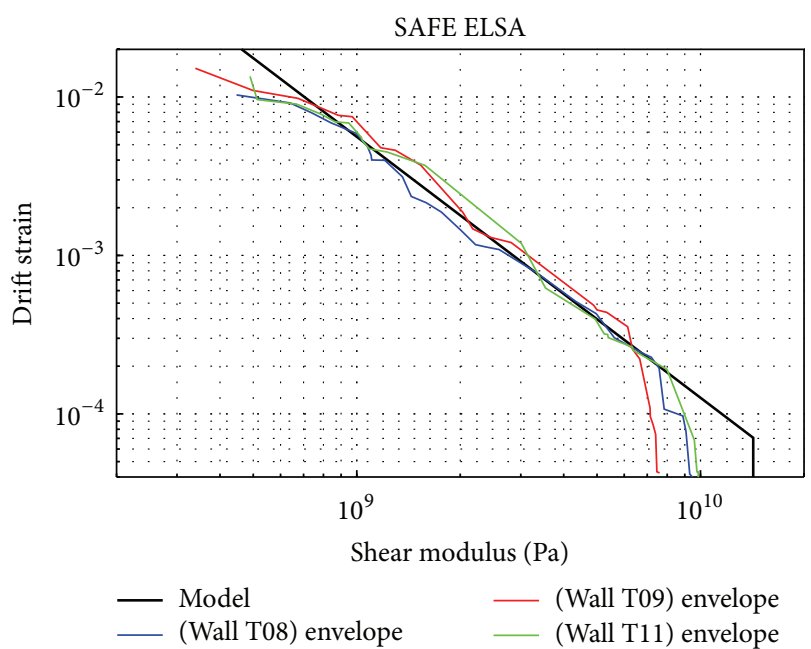

FIGURE 19: Identification of a unique model by including the data of walls T08, T09, and T11 (group “ $\rho=0.4$ ”).

which is the drift capacity at a reference "near-collapse" modulus $G_{B}$. By looking at Figure 23, we have adopted the following fixed values of the reference modulus:

$$
\begin{aligned}
G_{A} & =6.0 \cdot 10^{9} \mathrm{~Pa}, \\
G_{B} & =1.0 \cdot 10^{9} \mathrm{~Pa}
\end{aligned}
$$

in order to have them within the experimental RDL for all the walls but close to the ends of it.

Note that, according to the model, if a wall has one of these characteristic drift values higher, this means that for the corresponding level of damage (initial or near-collapse 


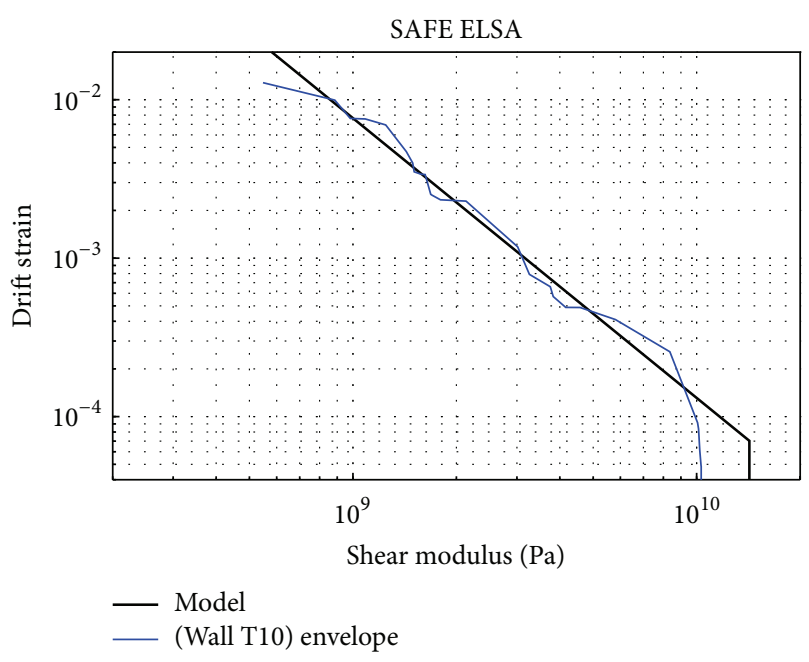

FIGURE 20: Identification of a unique model by including the data of wall T10 (group “ $\rho=0.6$ ”).

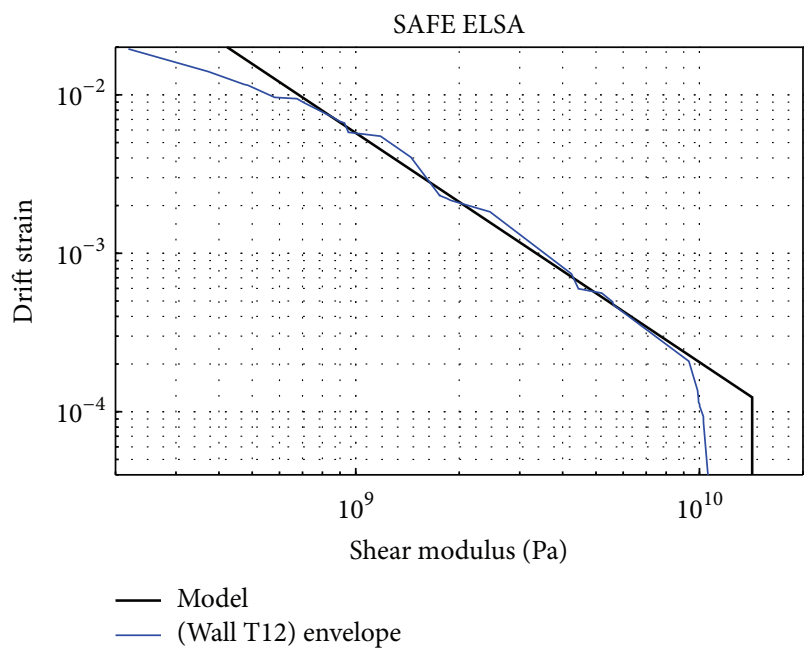

FIGURE 21: Identification of a unique model by including the data of wall T12 (group “ $\rho=0.11 ”)$.

modulus) there is a higher capacity of deformation and, consequently according to the model, a higher PGA earthquake can be resisted without new damage by that wall.

For every single wall, by using (47), (48), and (49) and the model parameters from Table 4 , the corresponding values of the characteristic capacity drifts have been computed and displayed in Table 6. Table 6 also contains for completeness the average between horizontal and vertical actually implemented value of reinforcement density as well as the vertical normal stress taken out of Tables 1 and 2.

In Figure 25 the model characteristic drifts $\gamma_{A}$ and $\gamma_{B}$ that define each one of those models listed in Table 6 are used as ordinates (in logarithmic scale), whereas the reinforcement density of every wall is used as abscissa (in linear scale). The walls tested with lower normal load (between 0.32 and $0.37 \mathrm{MPa}$ ) are represented by blue circles and the ones tested with higher normal load (1.01 MPa) are represented by red circles. The wall with added CFRP reinforcement

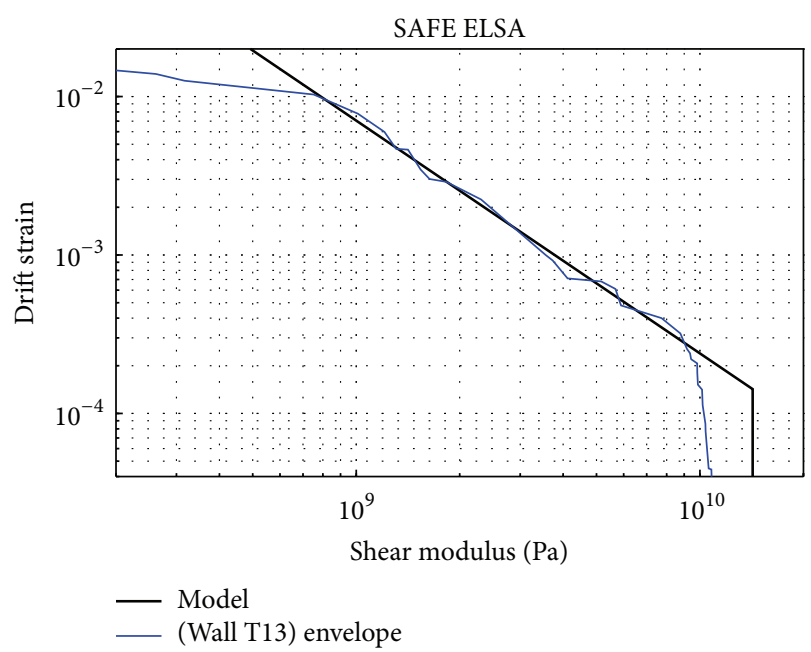

FIGURE 22: Identification of a unique model by including the data of wall T13 (group “CFRP”).

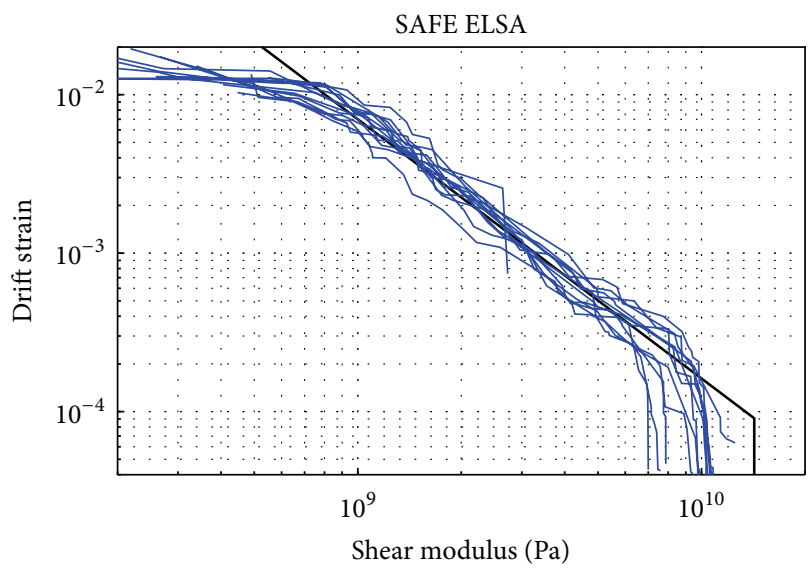

FIGURE 23: Identification of a unique model (thick black line) by including the data of walls T01 to T13 (group “ALL”).

(T13) is represented by a green circle. The purpose of these graphs in Figure 25 is to illustrate the qualitative effects of reinforcement and normal load on the characteristic drift values as follows:

(i) Point $A$, Figure 25(a): since the first part of the envelope is almost vertical, corresponding to elastic response of the noncracked wall, $\gamma_{A}$ should be closely related to the first cracking (degradation) phenomenon. As expected, this phenomenon is delayed (higher $\gamma_{A}$ ) by either a higher vertical stress (red circles) or an artificially increased tensile capacity (CFRP effect represented by the green circle). Otherwise, it should be kept in mind that, in reinforced concrete, the reinforcement should not play an actual role before the concrete is cracked. Therefore, as expected, $\gamma_{A}$ is not sensitive to the reinforcement ratio as confirmed by the graph. The observed dispersion could be due to the existing variability in the tension capacity among the specimens. 
TABLE 6: For every wall specimen, identified parameters of RDL (copied from Table 4) and derived characteristic capacity drifts $\gamma_{A}$ and $\gamma_{B}$.

\begin{tabular}{|c|c|c|c|c|c|c|}
\hline Wall & $\rho$ (reinf. dens. \%) & $\sigma_{n}(\mathrm{MPa})$ & $m$ & $\gamma_{R}(* 1 E-6)$ & $\gamma_{A}(* 1 E-6)$ & $\gamma_{B}(* 1 E-6)$ \\
\hline T01 & 0.770 & 0.37 & -1.7962 & 62 & 293 & 7312 \\
\hline T02 & 0.770 & 0.37 & -1.7593 & 73 & 332 & 7775 \\
\hline T03 & 0.770 & 0.37 & -1.7804 & 66 & 308 & 7479 \\
\hline T04 & 0.785 & 0.37 & -1.8360 & 78 & 383 & 10283 \\
\hline T05 & 0.767 & 0.32 & -1.7216 & 85 & 376 & 8230 \\
\hline T06 & 0.515 & 1.01 & -1.5513 & 125 & 477 & 7685 \\
\hline T07 & 0.515 & 1.01 & -1.6183 & 115 & 467 & 8482 \\
\hline T08 & 0.402 & 0.32 & -1.6039 & 70 & 279 & 4945 \\
\hline T09 & 0.402 & 0.32 & -1.6691 & 77 & 327 & 6515 \\
\hline $\mathrm{T} 10$ & 0.628 & 0.32 & -1.7660 & 70 & 324 & 7658 \\
\hline T11 & 0.402 & 0.32 & -1.7113 & 63 & 277 & 5947 \\
\hline $\mathrm{T} 12$ & 0.113 & 1.01 & -1.4469 & 123 & 431 & 5755 \\
\hline $\mathrm{T} 13$ & $0.402+$ CFRP & 0.32 & -1.4699 & 142 & 507 & 7059 \\
\hline
\end{tabular}

(ii) Point $B$, Figure 25(b): on the other hand, the ultimate shear capacity of a wall should be highly dependent on the reinforcement ratio. Effectively, (b) reveals that the associated drift value $\gamma_{B}$ is an increasing function of the reinforcement. A light positive effect can be also attributed to the normal load and very marginally to the external reinforcement with CFRP. Regarding the effect of the vertical load, we want to point out here that, in the spirit of the design rules presented by Labbé et al. [1], a lower vertical reinforcement ratio in walls T06 and T07 could be compensated by a larger vertical stress, making them comparable to T10 in terms of shear capacity. Then, as a confirmation, it is interesting to observe in Table 6 and in Figure 25(b) that these three specimens actually exhibit comparable $\gamma_{B}$ values.

3.7. Effect of the Reinforcement and the Normal Load on Crack Width. In order to assess in this section the effects on the crack width, the identified characteristic values of drift for the tested walls (previous section) will be converted into correlated crack width. This will be done by using the formula

$$
w=(0.1+133 \gamma) C(\rho)
$$

where $w$ is the magnitude of the crack width in mm, $\gamma$ is the drift in rad, and

$$
C(\rho)=0.2+\frac{0.9}{\rho+0.5}
$$

is the term that considers the effect of the average reinforcement density $\rho$ in \%. These empirical relations (50) and (51) were identified by Labbé et al. [1] from the crack width measurements recorded during the tests of SAFE. Thus, by applying these expressions (50) and (51) to each one of the tested walls (excluding Wall T13 which is not validated for these formulas), the associated crack width capacities

$$
w_{A}=\left(0.1+133 \gamma_{A}\right) C(\rho)
$$

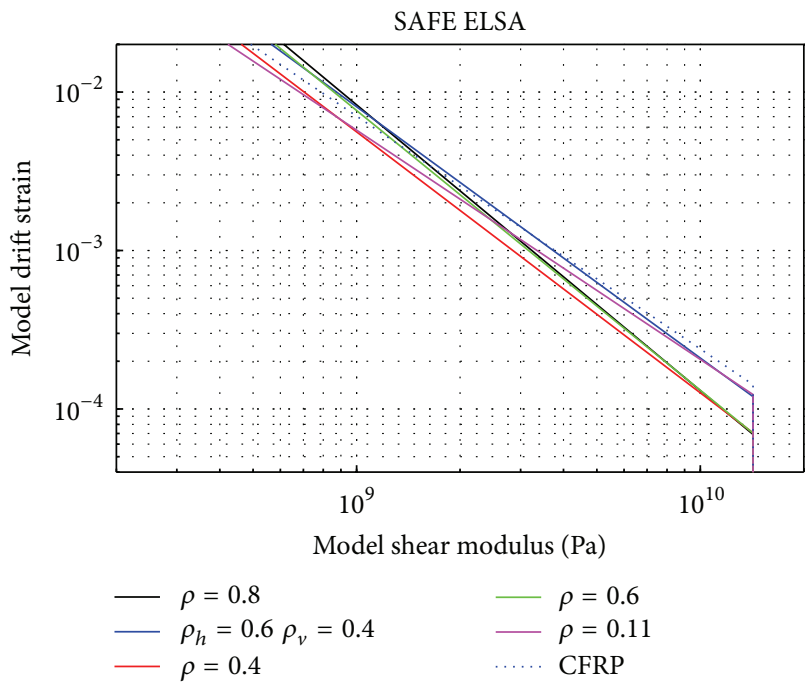

FIGURE 24: Comparison among the identified models for different groups.

at the reference initial modulus $G_{A}$ and

$$
w_{B}=\left(0.1+133 \gamma_{B}\right) C(\rho)
$$

at the reference near-collapse modulus $G_{B}$ can be computed, where $\gamma_{A}$ and $\gamma_{B}$ are taken from Table 6 . The computed values of these characteristic crack widths are displayed in Table 7 together with the actual values of average reinforcement density and normal load for the walls.

Now, as in the previous section, in Figure 26 the computed characteristic values are represented in logarithmic scale as a function of the reinforcement density and marked with different colours the different cases. The obtained graphs in Figure 26, together with the previous ones in Figure 25, can be interpreted as follows:

(i) Point $A$, Figure 26(a): once the cracking phenomenon has appeared, it is well known that the crack spacing 


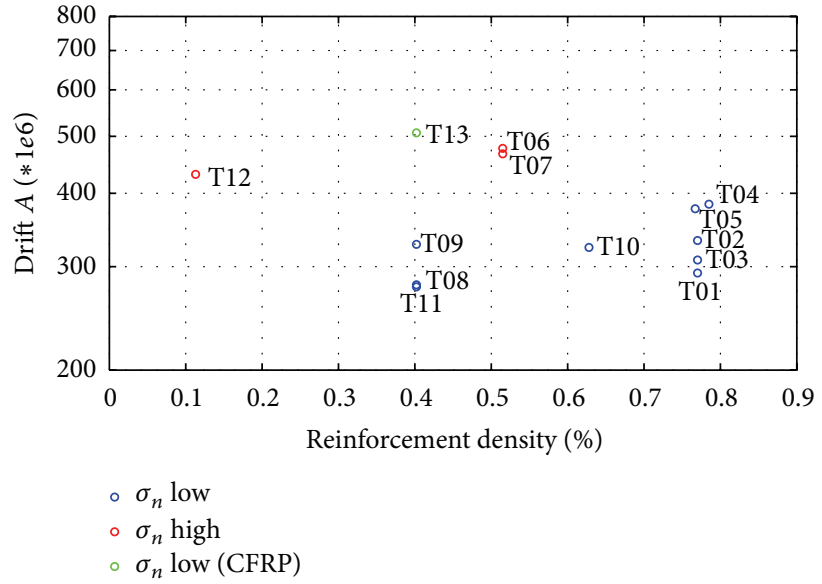

(a)

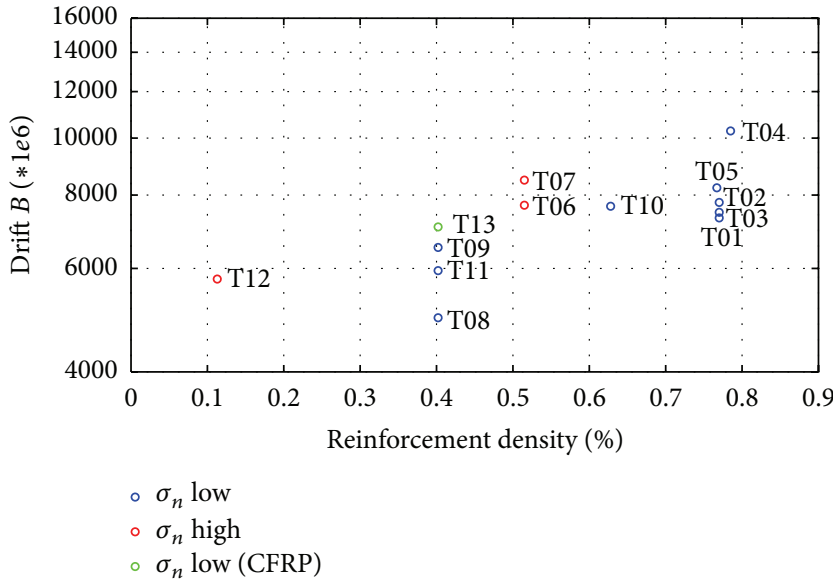

(b)

FIGURE 25: Comparison of the identified characteristic drifts for all the walls as a function of reinforcement and normal load. Drift $A$ ( $\gamma_{A}$, (a)) and Drift $B\left(\gamma_{B},(b)\right)$ are, respectively, the characteristic values at a reference initial stiffness and at a reference near-collapse stiffness.

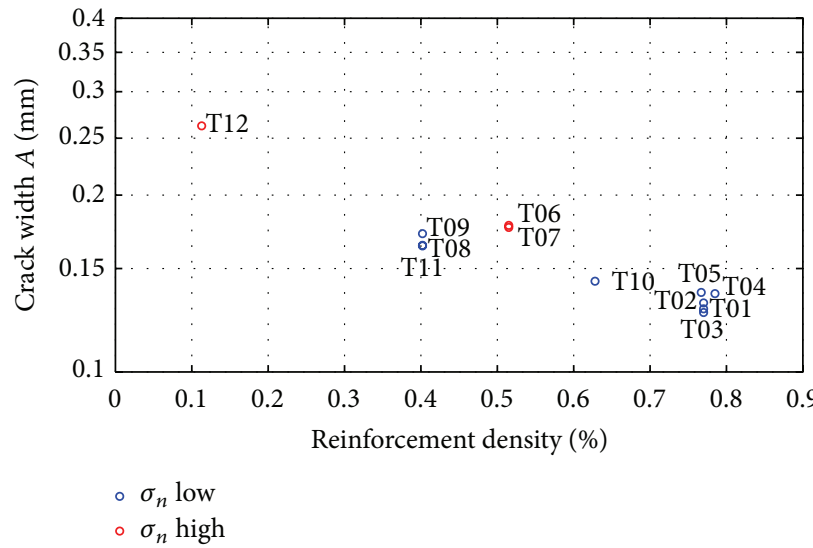

(a)

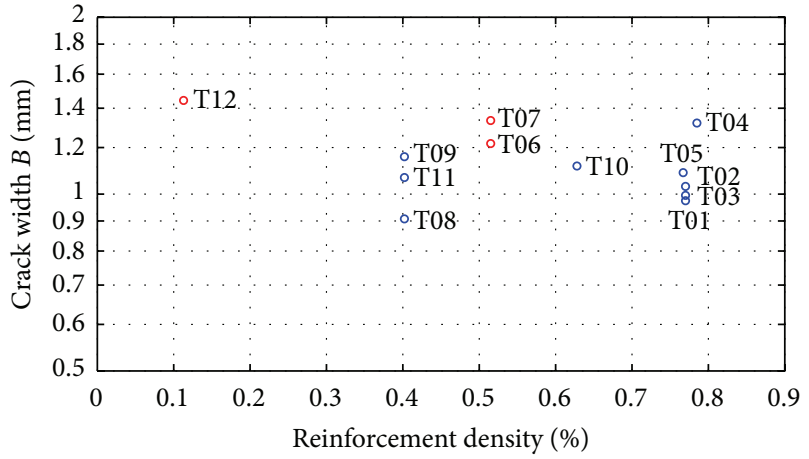

$$
\begin{array}{ll}
\text { - } \sigma_{n} \text { low } \\
\text { - } \sigma_{n} \text { high }
\end{array}
$$

(b)

FIGURE 26: Comparison of the identified characteristic crack widths for all the walls as a function of reinforcement and normal load. Crack width $A\left(w_{A},(\mathrm{a})\right)$ and Crack width $B\left(w_{B},(\mathrm{~b})\right)$ are, respectively, the characteristic values at a reference initial stiffness and at a reference near-collapse stiffness.

and crack width are sensitive to the reinforcement (the product of these two values being controlled by the drift). As expected, the larger the reinforcement is, the smaller the crack width develops (either for red and blue dots). Additional analyses would be necessary to discuss crack widths observed in the SAFE walls in comparison with formulas of the building codes and with the state of the art [15].

(ii) Point $B$, Figure 26(b): it is hard to recognise an effect of the reinforcement or the vertical stress on $w_{B}$, meaning basically that, for all the walls, Point $B$ corresponds to a certain common level of crack width, which can be associated just with the degraded shear modulus $G_{B}$ selected for this point.

3.8. Additional Remarks. The first remark concerns a more general application of the model described here. Independently of the practical reasons explained in Section 3.3, the choice, according to (40), of the corner of the polygonal line model at such a high stiffness as the one given by the design modulus would not be appropriate for the prediction of the response to nonsevere earthquakes on a virgin wall. This can be seen in all the figures comparing the actual envelopes with the corresponding model. For such purpose, alternatively, a value such as

$$
G_{L}=1.0 \cdot 10^{10} \mathrm{~Pa}
$$

can be recommended having into account the observations reported by Labbé et al. [1] regarding the average initial modulus found to be around 0.67 times the design modulus. Even though the reproducibility of the experimental envelope at this corner point was not high and the model predictions cannot be very reliable in that range of deformation, the latter choice of this corner modulus (54) does change the earthquake response predictions for the experiments regarding specimens T12 and T13. This effect is strong and it is 
TABLE 7: For every wall specimen, derived characteristic crack widths $w_{A}$ and $w_{B}$.

\begin{tabular}{lccccc}
\hline Wall & $\rho$ (reinf. dens. \%) & $\sigma_{n}$ & $C(\rho)$ & $w_{A}(\mathrm{~mm})$ & $w_{B}(\mathrm{~mm})$ \\
\hline T01 & 0.770 & 0.37 & 0.91 & 0.13 & 0.97 \\
T02 & 0.770 & 0.37 & 0.91 & 0.13 & 1.03 \\
T03 & 0.770 & 0.37 & 0.91 & 0.13 & 0.99 \\
T04 & 0.785 & 0.37 & 0.90 & 0.14 & 1.32 \\
T05 & 0.767 & 0.32 & 0.91 & 0.14 & 1.09 \\
T06 & 0.515 & 1.01 & 1.09 & 0.18 & 1.22 \\
T07 & 0.515 & 1.01 & 1.09 & 0.18 & 1.33 \\
T08 & 0.402 & 0.32 & 1.20 & 0.16 & 0.91 \\
T09 & 0.402 & 0.32 & 1.20 & 0.17 & 1.16 \\
T10 & 0.628 & 0.32 & 1.00 & 0.14 & 1.12 \\
T11 & 0.402 & 0.32 & 1.20 & 0.16 & 1.07 \\
T12 & 0.113 & 1.01 & 1.67 & 0.26 & 1.44 \\
\hline
\end{tabular}

reflected in the alternative values of the prediction error at the corresponding rows in Tables 4 and 5. Indeed the prediction error diminishes for these two walls by using the reduced initial modulus (54) in the model. The reason for which relatively lower intensity earthquakes were applied to these walls, and not for the other ones, could apparently be that their adopted design was too conservative. Particularly, for the case of T12 with a relatively low steel density, Labbé et al. [1] already mention that, by disregarding the tensile strength of the concrete in the formula of the design strength (11), the wall capacity might be underestimated. On the other hand, for the case of T13, the presence of the CFRP, which was not considered in its design strength parameter, could also justify this phenomenon.

The second remark concerns the onset of damage. In principle, the first crack appears when the tensile capacity of the concrete is exceeded. The Eurocode 2 rule to derive the tensile capacity from the compression capacity is

$$
\left.f_{t 28}=0.3\left(f_{c 28}\right)^{2 / 3} \quad \text { in } \mathrm{MPa}\right) .
$$

The individual $f_{c 28}$ of walls T03 to T12 as presented by Labbé et al. [1], showing a noticeable variability, when converted to tension capacity through this formula (55), lead to an average value

$$
\overline{f_{t 28}}=3.6 \mathrm{MPa} \text {. }
$$

Now, with the average initial shear modulus value (54) and assuming a nil vertical stress, it leads to a drift strain of 3.6 . $10^{-4}$. In the case of pure elastic behaviour in shear without any cracking, disregarding the reinforcement, this value should deliver an average approximation of the lower corner drift $\gamma_{L}$. However, it is normally reached by the envelopes shown in Figure 23 only after some degradation is already present. In average the vertical part of the envelope terminates rather at around $2 \cdot 10^{-4}$. This fact could be regarded as an indication that some degradation may develop in the walls before the first crack reaches it limit stress and presumably becomes visible.

\section{Conclusions}

The SAFE program consisted on seismic PsD tests on $13 \mathrm{RC}$ shear wall specimens submitted to the same accelerogram for several increasing intensities. The parameters that changed among the walls were the thickness, the reinforcement, the normal load, and the design frequency, which was controlled by the theoretical mass introduced in the equation of motion for running the PsD tests. In fact, the design frequency was observed to have a strong influence on the damage at the respective design intensity of the wall and on the safety margin for higher intensities [1]. Some of the aspects that interact to produce this effect are made clearer by the current study.

The proposed model is based on identification methods applied to the observed experimental response. The most important identified parameters at every time instant are the secant stiffness, coming from the Spatial Model, and the displacement oscillation amplitude, coming from the Hilbert Transform. The trajectory of these two variables, or equivalently of the shear modulus and the drift amplitude, shows a clear envelope during the successive earthquakes on the same wall. This envelope can be associated with the idea that the stiffness remains roughly constant until new damage is introduced by reaching a displacement amplitude that is larger than any amplitude experienced before. Based on this idea that was already present in some models available in the literature, the envelope line computed from the identified variables for a tested wall can be interpreted as a capacity line that is able to predict response frequency values associated with displacement oscillation amplitudes or vice versa.

Moreover, the intersection of such capacity line with the demand response spectrum of an applied earthquake predicts the response maximum displacement and associated frequency. Further on, in the double logarithmic representation for the chosen variables of stiffness and deformation, we propose to substitute the experimentally derived envelope by a piecewise line curve, with a main straight branch called Regular Degradation Line (RDL). Another two branches with different slope could be required for the very small or very large deformations, but the reproducibility of the experimental data was not so high in these areas.

It is shown that the RDL identified for every wall is able to predict the maximum response to the applied earthquakes with standard error of $25 \%$, average among the different walls, when using the response spectrum based on $5 \%$ damping ratio. If the RDL of the specific wall is replaced by a common RDL for all the walls (disregarding the different reinforcement and normal load of each one), the standard error is $40 \%$. Other values of damping ratio from $4 \%$ to $7 \%$ have been found to give worse global results. Alternatively, identified values of instantaneous damping identified by the Spatial Model have been considered in this study but for the moment they do not lead to better general interpretations.

For walls of the SAFE program with the same reinforcement and normal load, very similar experimental envelope was derived and a unique RDL can be used to model the whole group, even if the different walls inside the group had been tested with different assigned design frequency. Then it is realized that the different positioning of the design 
frequency with respect to the spectrum, changes, not only the computed design intensity (that was based on a constant response frequency method), but also very drastically the design margins predicted according to the current model. The effect is directly related to the closeness of the local slope of the spectrum to the slope of the RDL. For the adopted spectrum within the SAFE program, the slope is always decreasing with the frequency and becoming closer to the slope of the capacity line. This is translated in shorter safety margins for the cases with larger assigned design frequency as it had been observed from the experiments and is now explained by the model.

By analysing the changes on the identified RDL model with respect to the different parameters of the walls, some effects can be recognised. Particularly, for the tested walls in the SAFE program, the characteristic drift at the initial shear modulus state is mostly dominated by the added normal load and the presence of the external CFRP, whereas the characteristic drift at the near-collapse state is mostly determined by the different amounts of steel reinforcement.

By using the empirical correlation between drift and crack width that was proposed in a previous study, the effects can also be assessed on the crack width. It is observed that at the initial shear modulus state, the crack width is smaller for the walls with higher reinforcement, whereas at the near-collapse state the crack width does not show a clear correlation with the amount of reinforcement.

\section{Competing Interests}

The authors declare that they have no competing interests.

\section{Acknowledgments}

The experimental program SAFE was financially supported by Electricité de France and COGEMA and executed at the ELSA laboratory of the JRC of the European Commission. Many colleagues from the collaborating institutions contributed to the quality of the results that has made possible several studies including the current one. From the ELSA laboratory we are particularly grateful to the contributions of Guido Verzeletti, Georges Magonette, and Vito Renda.

\section{References}

[1] P. Labbé, P. Pegon, J. Molina, C. Gallois, and D. Chauvel, "The SAFE experimental research on the frequency dependence of shear wall seismic design margins," Journal of Earthquake Engineering, vol. 20, no. 1, pp. 101-128, 2016.

[2] D. Benedetti and M. P. Limongelli, "A model to estimate the virgin and ultimate effective stiffnesses from the response of a damaged structure to a single earthquake," Earthquake Engineering and Structural Dynamics, vol. 25, no. 10, pp. 1095-1108, 1996.

[3] M. Brun, J. M. Reynouard, and L. Jezequel, "A simple shear wall model taking into account stiffness degradation," Engineering Structures, vol. 25, no. 1, pp. 1-9, 2003.

[4] M. Brun, P. Labbe, D. Bertrand, and A. Courtois, "Pseudodynamic tests on low-rise shear walls and simplified model based on the structural frequency drift," Engineering Structures, vol. 33, no. 3, pp. 796-812, 2011.

[5] P. Fajfar, "Capacity spectrum method based on inelastic demand spectra," Earthquake Engineering \& Structural Dynamics, vol. 28, no. 9, pp. 979-993, 1999.

[6] P. B. Labbé, "Categorization of seismically-induced stresses for civil and mechanical engineering," Nuclear Engineering and Design, vol. 255, pp. 240-247, 2013.

[7] P. Pegon, G. Magonette, F. J. Molina et al., "Programme SAFE: rapport du test T4," Note Technique du CCR no. I.98.32, 1998.

[8] P. Pegon, F. J. Molina, and G. Magonette, "Continuous pseudodynamic testing at ELSA," in Hybrid Simulation; Theory, Implementation and Applications, V. E. Saouma and M. V. Sivaselvan, Eds., pp. 79-88, Taylor \& Francis/Balkema, 2008.

[9] F. J. Molina, G. Magonette, P. Pegon, and B. Zapico, "Monitoring damping in pseudo-dynamic tests," Journal of Earthquake Engineering, vol. 15, no. 6, pp. 877-900, 2011.

[10] F. J. Molina, F. Marazzi, B. Viaccoz, and A. Bosi, "Stability and accuracy in a hybrid test example," Earthquake Engineering and Structural Dynamics, vol. 42, no. 3, pp. 469-475, 2013.

[11] F. J. Molina, P. Pegon, and G. Verzeletti, “Time-domain identification from seismic pseudodynamic test results on civil engineering specimens," in Proceedings of the 2nd International Conference on Identification in Engineering Systems, The Cromwell Press, 1999.

[12] F. J. Molina, G. Verzeletti, P. Pegon et al., Ensayos Pseudodinámicos a Cortante en Muros de Hormigón Armado. Evolución de la Frecuencia y el Amortiguamiento durante el Terremoto, Primer Congreso Nacional de Ingeniería Sísmica, AEIS, Murcia, Spain, 1999.

[13] F. J. Molina, Spatial and Filter Models, MATLAB Functions, Matworks File Exchange, The MathWorks, Natick, Mass, USA, 2011, http://www.mathworks.com/matlabcentral/fileexchange/32634.

[14] H. Herlufsen, "Dual channel FFT analysis (Part II)," Brüel \& Kjaer Technical Reviews 2-1984, 1984.

[15] E. Gallitre, Fissuration des voiles courts en béton armé soumis à des solicitations sismiques, evaluation des ouvertures de fissures [Ph.D. thesis], Institut National des Sciences Appliquées, Lyon, France, 2008. 

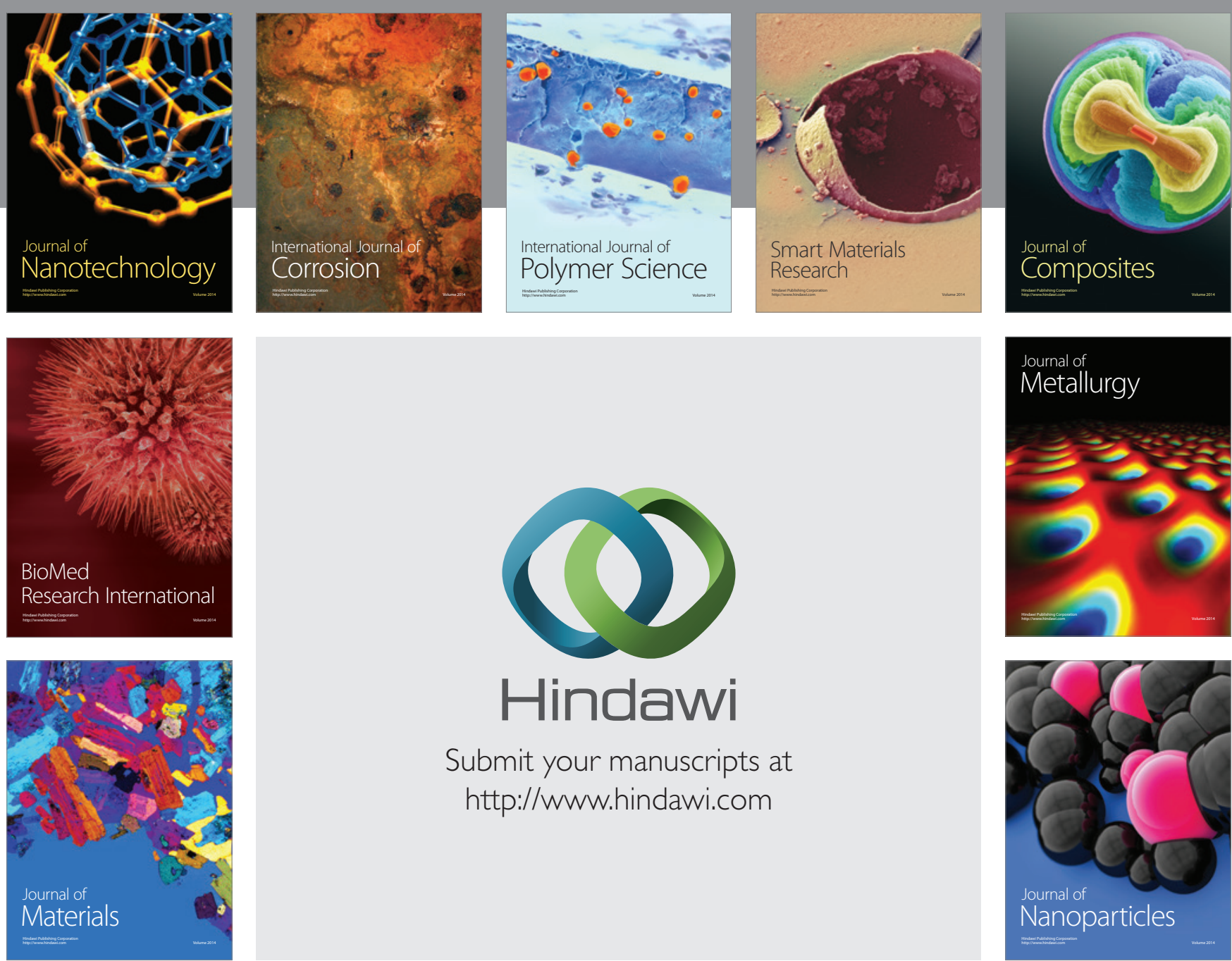

\section{Hindawi}

Submit your manuscripts at

http://www.hindawi.com

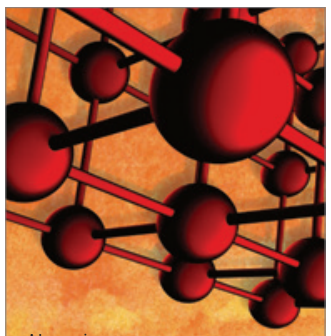

Materials Science and Engineering
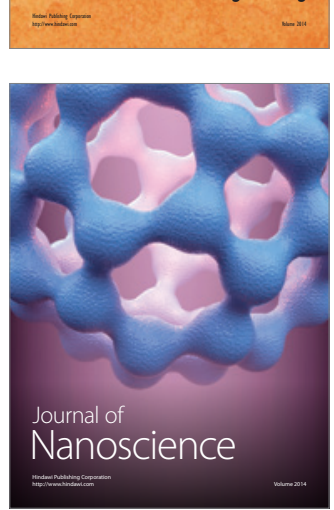
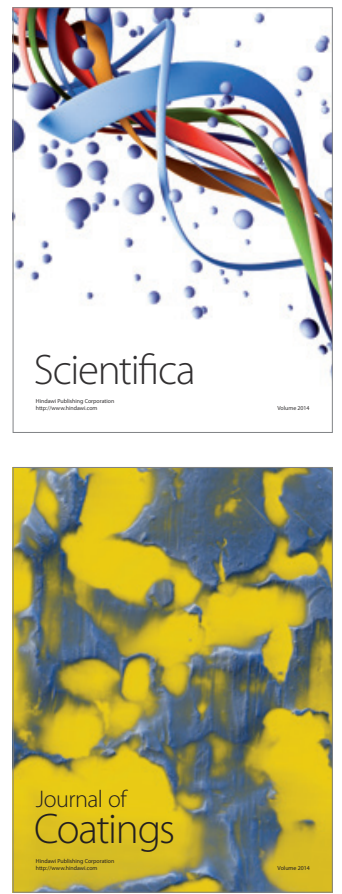
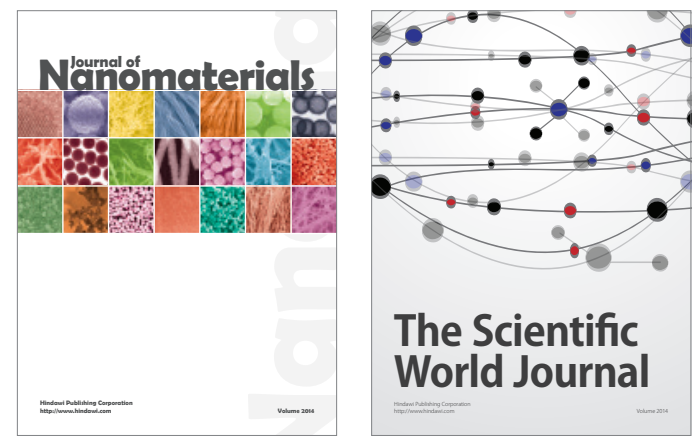

The Scientific World Journal
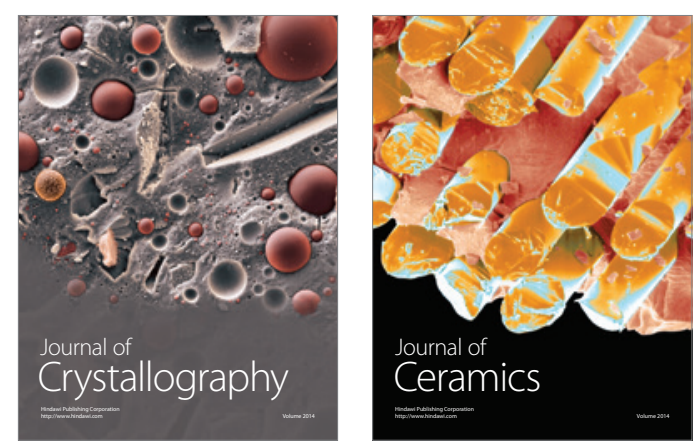
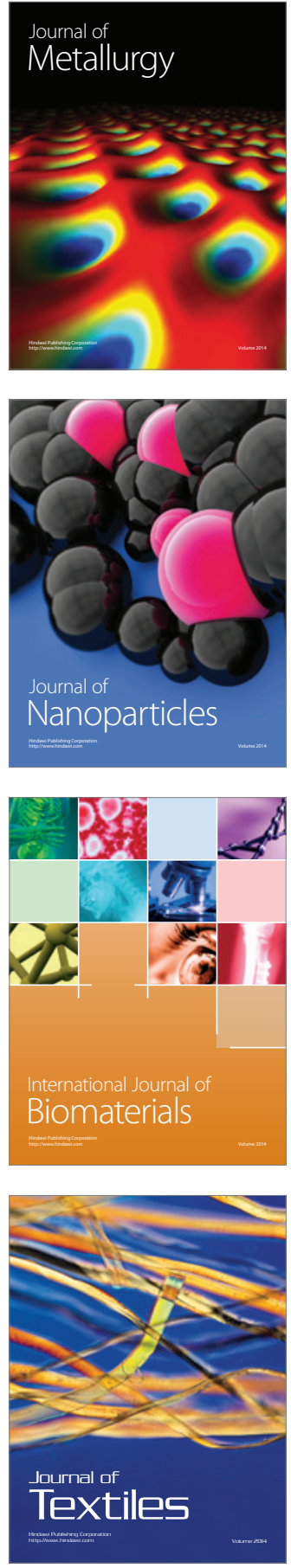